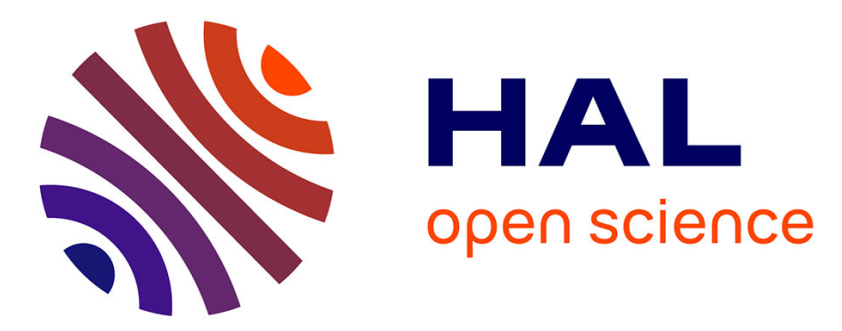

\title{
Polynomial-degree-robust a posteriori estimates in a unified setting for conforming, nonconforming, discontinuous Galerkin, and mixed discretizations
}

Alexandre Ern, Martin Vohralík

\section{> To cite this version:}

Alexandre Ern, Martin Vohralík. Polynomial-degree-robust a posteriori estimates in a unified setting for conforming, nonconforming, discontinuous Galerkin, and mixed discretizations. SIAM Journal on Numerical Analysis, 2015, 53 (2), pp.1058-1081. 10.1137/130950100 . hal-00921583v2

\section{HAL Id: hal-00921583 \\ https://inria.hal.science/hal-00921583v2}

Submitted on 30 Jul 2014

HAL is a multi-disciplinary open access archive for the deposit and dissemination of scientific research documents, whether they are published or not. The documents may come from teaching and research institutions in France or abroad, or from public or private research centers.
L'archive ouverte pluridisciplinaire HAL, est destinée au dépôt et à la diffusion de documents scientifiques de niveau recherche, publiés ou non, émanant des établissements d'enseignement et de recherche français ou étrangers, des laboratoires publics ou privés.

\section{(c)(1)}

Distributed under a Creative Commons Attribution| 4.0 International License 


\title{
Polynomial-degree-robust a posteriori estimates in a unified setting for conforming, nonconforming, discontinuous Galerkin, and mixed discretizations*
}

\author{
Alexandre Ern ${ }^{\dagger} \quad$ Martin Vohralík $k^{\ddagger}$
}

July 30,2014

\begin{abstract}
We present equilibrated flux a posteriori error estimates in a unified setting for conforming, nonconforming, discontinuous Galerkin, and mixed finite element discretizations of the two-dimensional Poisson problem. Relying on the equilibration by mixed finite element solution of patchwise Neumann problems, the estimates are guaranteed, locally computable, locally efficient, and robust with respect to polynomial degree. Maximal local overestimation is guaranteed as well. Numerical experiments suggest asymptotic exactness for the incomplete interior penalty discontinuous Galerkin scheme.
\end{abstract}

Key words: a posteriori error estimate, equilibrated flux, unified framework, robustness, polynomial degree, conforming finite element method, nonconforming finite element method, discontinuous Galerkin method, mixed finite element method

\section{Introduction}

A posteriori error estimates in the conforming finite element setting have already received a large attention. In particular, following the concept of Prager and Synge [64], cf. also Synge [72], Aubin and Burchard [13], and Hlaváček et al. [50], and invoking fluxes in the $\mathbf{H}(\operatorname{div}, \Omega)$ space, guaranteed upper bounds on the error can be obtained. A general functional framework delivering guaranteed upper bounds, independent of the numerical method, has been derived by Repin [66, 67, 68]. It does not rely on Galerkin orthogonality neither on local equilibration and accommodates an arbitrary flux reconstruction. The idea of using a local residual equilibration procedure for the normal face fluxes reconstruction has been proposed by Ladevèze [55], Ladevèze and Leguillon [56], Kelly [51], Ainsworth and Oden [7, 8], and Parés et al. [61, 62]. In this context, guaranteed upper bounds typically require solving infinite-dimensional element problems, which, in practice, are approximated. On the other hand, an essential property achieved by means of local equilibration procedures is local efficiency, meaning that the derived estimators also represent local lower bounds of the error, up to a generic constant. This appears to be crucial in view of local mesh refinement, as well as in order to obtain robustness in singularly perturbed problems. Cheap local flux equilibrations leading to a fully computable guaranteed upper bound have been obtained by Destuynder and Métivet [38]. Later, mixed finite element solutions of local Neumann problems posed over patches of (sub)elements, where one minimizes locally the estimator contributions, were proposed, see Luce and Wohlmuth [57], Braess and Schöberl [19], and [77, 30, 79]. As a matter of fact, lifting the normal face fluxes of the equilibrated residual method to $\mathbf{H}(\operatorname{div}, \Omega)$ immediately yields equilibrated fluxes, cf. Nicaise et al. [59]. Then, both a guaranteed bound and local efficiency are obtained. For computational comparisons of some of these approaches in the lowest-order case, see Carstensen and Merdon [28].

The theory in the nonconforming setting, where the discrete solution (potential) is not in the energy space $H^{1}(\Omega)$, appears to be less developed. First contributions are those of Agouzal [1] and Dari et al.

\footnotetext{
*This work was supported by the ERC-CZ project MORE "MOdelling REvisited + MOdel REduction" LL1202.

†Université Paris-Est, CERMICS (ENPC), 77455 Marne-la-Vallée cedex 2, France (ern@cermics.enpc.fr).

‡INRIA Paris-Rocquencourt, B.P. 105, 78153 Le Chesnay, France (martin.vohralik@inria.fr).
} 
[35], whereas a guaranteed error upper bound in the lowest-order Crouzeix-Raviart case can be obtained along the lines of Destuynder and Métivet [37], see Ainsworth [2], Kim [52, 53], or [76]. Different flux equilibrations exist and tight links hold between them, see [46]. Higher-order methods have been treated by Ainsworth and Rankin [9], and a survey and a computational comparison in the lowest-order case can be found in Carstensen and Merdon [29]. For the discontinuous Galerkin method, first guaranteed upper bounds by locally equilibrated fluxes and $H^{1}(\Omega)$-conforming reconstructed potentials have been obtained by Ainsworth [3], Kim [52, 53], Cochez-Dhondt and Nicaise [32], Ainsworth and Rankin [10], and [41, 43, 42], see also the references therein. Similar results for mixed finite elements can be found in Kim [52, 54], Ainsworth [4], Ainsworth and Ma [6], and [76, 78]. All the cited references typically prove local efficiency as well.

When the flux equilibration is achieved by mixed finite element solutions of local Neumann problems, the local efficiency result, in the conforming finite element setting, can be sharpened by showing that the efficiency constant is independent of the underlying polynomial degree. This important result was recently proven by Braess et al. [18], and we refer to it as polynomial-degree robustness. This robustness property stands in contrast to the class of usual residual-based estimators, cf. Verfürth [74], which yield local efficiency, but where such a robustness does not hold, see Melenk and Wohlmuth [58]. The first key ingredient for the proof in [18] are continuous-level problems on patches of elements around vertices featuring the hat functions, similar to those considered already in Carstensen and Funken [24]. This replaces the usual bubble function technique. The second key ingredient is the polynomial-degree-robust stability of mixed finite elements of [18, Theorem 7], hinging on the polynomial-degree robust elementwise construction of a right inverse of the divergence operator in polynomial spaces by Costabel and McIntosh [34] and on the polynomial extension operators by Demkowicz et al. [36].

We finally mention that unified frameworks for different discretization methods have been conceived recently, see Carstensen et al. [22, 27, 26, 23], Ainsworth [5], and, using the equilibrated fluxes, in [44, 49, 45].

In the present paper, we unify the potentials and equilibrated fluxes approach for most standard discretization schemes, including conforming, nonconforming (where the potential interface jumps satisfy some orthogonality conditions), discontinuous Galerkin, and mixed finite elements. The construction of the estimators becomes method independent, being close to that of Destuynder and Métivet [38] and coinciding with that of Braess and Schöberl [19] for fluxes in the conforming case, while being closely related to that of Carstensen and Merdon [29] for potentials in the nonconforming case. In the discontinuous Galerkin and mixed finite element cases, such an approach appears to be new. The potentials and fluxes are actually constructed by the same patchwise problems with different right-hand sides in the present two-dimensional setting. Most importantly, we prove the polynomial-degree robustness in this unified setting comprising all the discussed discretization schemes. Moreover, we can also guarantee a maximal overestimation factor, a feature which can be important in optimal convergence proofs. Additionally, numerical experiments for the incomplete interior penalty discontinuous Galerkin scheme suggest asymptotic exactness.

The paper is organized as follows: The setting is described in Section 2. The main results together with their proofs are collected in Section 3. Applications to most standard numerical methods are showcased in Section 4, and a numerical illustration is presented in Section 5. Concluding remarks in Section 6 close the paper.

\section{Setting}

We start by introducing the continuous and discrete settings.

\subsection{Sobolev spaces}

Let $\Omega \subset \mathbb{R}^{2}$ be a polygonal domain (open, bounded, and connected set). We denote by $H^{1}(\Omega)$ the Sobolev space of $L^{2}(\Omega)$ functions with weak gradients in $\left[L^{2}(\Omega)\right]^{2}$ and by $H_{0}^{1}(\Omega)$ its zero-trace subspace. $\mathbf{H}(\operatorname{div}, \Omega)$ stands for the space of $\left[L^{2}(\Omega)\right]^{2}$ functions with weak divergences in $L^{2}(\Omega)$. The notations $\nabla$ and $\nabla$. are used respectively for the weak gradient and divergence. Let $\mathrm{R}_{\frac{\pi}{2}}:=\left(\begin{array}{cc}0 & -1 \\ 1 & 0\end{array}\right)$ be the matrix of rotation by $\frac{\pi}{2}$; then $\mathrm{R}_{\frac{\pi}{2}} \nabla$ stands for the weak curl, i.e., the rotated gradient: for $v \in H^{1}(\Omega), \mathrm{R}_{\frac{\pi}{2}} \nabla v=\left(-\partial_{y} v, \partial_{x} v\right)$. For a subdomain $\omega$ of $\Omega$, we denote by $(\cdot, \cdot)_{\omega}$ the $L^{2}(\omega)$-inner product, by $\|\cdot\|_{\omega}$ the associated norm (we omit the 
index when $\omega=\Omega$ ), and by $|\omega|$ the Lebesgue measure of $\omega$. For $\omega \subset \mathbb{R}^{1},\langle\cdot, \cdot\rangle_{\omega}$ stands for the 1-dimensional $L^{2}(\omega)$-inner product or for the appropriate duality pairing on $\omega$.

\subsection{Meshes}

We consider partitions $\mathcal{T}_{h}$ of $\Omega$ which consist either of closed triangles or of closed rectangles $K$ such that $\bar{\Omega}=\bigcup_{K \in \mathcal{T}_{h}} K$. We suppose that $\mathcal{T}_{h}$ is matching, i.e., such that for two distinct elements, their intersection is either an empty set or a common edge or a common vertex. For any $K \in \mathcal{T}_{h}, \mathbf{n}_{K}$ stands for the outward unit normal vector to $K$ and $h_{K}$ denotes the diameter of $K$. The edges of the mesh form the set $\mathcal{E}_{h}$ divided into interior edges $\mathcal{E}_{h}^{\text {int }}$ and boundary edges $\mathcal{E}_{h}^{\text {ext }}$. A generic edge is denoted by $e$ and its diameter by $h_{e}$. For any $e \in \mathcal{E}_{h}, \mathbf{n}_{e}$ stands for the unit normal vector to $e$; the orientation is arbitrary but fixed for $e \in \mathcal{E}_{h}^{\text {int }}$ and points outwards of $\Omega$ for $e \in \mathcal{E}_{h}^{\text {ext }}$. The set of vertices is denoted by $\mathcal{V}_{h}$; it is decomposed into interior vertices $\mathcal{V}_{h}^{\text {int }}$ and boundary vertices $\mathcal{V}_{h}^{\text {ext }}$. For $\mathbf{a} \in \mathcal{V}_{h}, \mathcal{T}_{\mathbf{a}}$ denotes the patch of the elements of $\mathcal{T}_{h}$ which share a and $\omega_{\mathbf{a}}$ the corresponding open subdomain of diameter $h_{\omega_{\mathbf{a}}}$. For $K \in \mathcal{T}_{h}, \mathcal{V}_{K}$ denotes the set of vertices of $K$. From Section 3.2 onwards, we will need the shape-regularity assumption requesting the existence of a constant $\kappa_{\mathcal{T}}>0$ such that $\max _{K \in \mathcal{T}_{h}} h_{K} / \varrho_{K} \leq \kappa_{\mathcal{T}}$ for all triangulations $\mathcal{T}_{h}$, with $\varrho_{K}$ being the diameter of the largest ball inscribed in $K$. We will also invoke the average operator $\{\cdot\}$ yielding the mean value of the traces from adjacent mesh elements on inner edges and the actual trace on boundary edges; similarly, the jump operator $\llbracket \rrbracket$ yields the difference evaluated along $\mathbf{n}_{e}$ on $e \in \mathcal{E}_{h}^{\text {int }}$ and the actual trace on $e \in \mathcal{E}_{h}^{\text {ext }}$.

\subsection{Broken spaces}

At some places, we will use the mesh-related broken Sobolev spaces $H^{1}\left(\mathcal{T}_{h}\right):=\left\{v \in L^{2}(\Omega) ;\left.v\right|_{K} \in H^{1}(K)\right.$ for all $\left.K \in \mathcal{T}_{h}\right\}$ as well as $\mathbf{H}\left(\operatorname{div}, \mathcal{T}_{h}\right):=\left\{\mathbf{v} \in\left[L^{2}(\Omega)\right]^{2} ;\left.\mathbf{v}\right|_{K} \in \mathbf{H}(\operatorname{div}, K)\right.$ for all $\left.K \in \mathcal{T}_{h}\right\}$. Then, $\nabla$ stands for the broken (elementwise) weak gradient, $\nabla$. for the broken (elementwise) weak divergence, and $\mathrm{R}_{\frac{\pi}{2}} \nabla$ for the broken (elementwise) weak curl.

\section{$2.4 \quad$ Finite element spaces}

We use $\mathbb{P}_{p}(K)$ (respectively, $\left.\mathbb{Q}_{p}(K)\right), p \geq 0$, to denote polynomials in $K \in \mathcal{T}_{h}$ of total degree at most $p$ (respectively, at most $p$ in each variable), and $\mathbb{P}_{p}\left(\mathcal{T}_{h}\right)$ and $\mathbb{Q}_{p}\left(\mathcal{T}_{h}\right)$ to denote the corresponding broken spaces. For a vertex $\mathbf{a} \in \mathcal{V}_{h}$, let $\psi_{\mathbf{a}}$ stand for the "hat" function from $\mathbb{P}_{p}\left(\mathcal{T}_{h}\right) \cap H^{1}(\Omega)$ or $\mathbb{Q}_{p}\left(\mathcal{T}_{h}\right) \cap H^{1}(\Omega)$ which takes value 1 at the vertex $\mathbf{a}$ and zero at the other vertices. Following Brezzi and Fortin [21] or Roberts and Thomas [69], let $\mathbf{R T}_{p}:=\left\{\mathbf{v}_{h} \in \mathbf{H}(\operatorname{div}, \Omega) ;\left.\mathbf{v}_{h}\right|_{K} \in \mathbf{R T}_{p}(K)\right\}, p \geq 0$, with the local spaces $\mathbf{R T}_{p}(K):=\left[\mathbb{P}_{p}(K)\right]^{2}+\mathbb{P}_{p}(K) \mathbf{x}$ on triangles and $\mathbf{R T}_{p}(K):=\mathbb{Q}_{p+1, p}(K) \times \mathbb{Q}_{p, p+1}(K)$ on rectangles, where $\mathbb{Q}$... $(K)$ sets the maximal polynomial degree separately for each variable. We will employ this RaviartThomas (RT) family, with $\mathbb{P}_{p}\left(\mathcal{T}_{h}\right)$ or $\mathbb{Q}_{p}\left(\mathcal{T}_{h}\right)$ for the corresponding $L^{2}(\Omega)$ approximations, and we use the abstract notation $\mathbf{V}_{h}:=\mathbf{R} \mathbf{T}_{p}, Q_{h}:=\mathbb{P}_{p}\left(\mathcal{T}_{h}\right)$ or $\mathbb{Q}_{p}\left(\mathcal{T}_{h}\right), \mathbf{V}_{h}(K):=\mathbf{R} \mathbf{T}_{p}(K)$, and $Q_{h}(K):=\mathbb{P}_{p}(K)$ or $\mathbb{Q}_{p}(K)$; this allows us to discuss other families, like the Brezzi-Douglas-Marini one in Remark 3.21.

\subsection{The model problem}

We study in this paper the Poisson problem for the Laplace equation: for $f \in L^{2}(\Omega)$, find $u$ such that

$$
\begin{aligned}
-\Delta u=f & \text { in } \Omega, \\
u=0 & \text { on } \partial \Omega .
\end{aligned}
$$

The weak formulation consists in finding $u \in H_{0}^{1}(\Omega)$ such that

$$
(\nabla u, \nabla v)=(f, v) \quad \forall v \in H_{0}^{1}(\Omega) .
$$

Existence and uniqueness of the solution $u$ to (2.2) follow from the Riesz representation theorem. We term the scalar-valued function $u$ the potential and the vector-valued function $\sigma:=-\nabla u$ the flux. Extensions to inhomogeneous Dirichlet and Neumann boundary conditions, more general meshes, meshes with hanging nodes, and approximations with varying polynomial degree are possible modulo necessary technicalities. 


\section{$3 \quad$ Main results}

We present in this section our main results. The guaranteed error upper bound is presented in Section 3.1 and a lower bound robust with respect to the polynomial degree is stated in Section 3.2. Maximal overestimation is investigated in Section 3.3.

\subsection{Guaranteed reliability}

Let $u_{h}$ denote the given approximate solution to problem $(2.2)$. In this section, we only need $u_{h} \in H^{1}\left(\mathcal{T}_{h}\right)$.

\subsubsection{Equilibrated flux and potential reconstructions}

Discrete solutions are typically such that $u_{h} \notin H_{0}^{1}(\Omega),-\nabla u_{h} \notin \mathbf{H}(\operatorname{div}, \Omega)$, or $\nabla \cdot\left(-\nabla u_{h}\right) \neq f$, while the weak solution satisfies $u \in H_{0}^{1}(\Omega), \boldsymbol{\sigma} \in \mathbf{H}(\operatorname{div}, \Omega)$, and $\nabla \cdot \boldsymbol{\sigma}=f$ with $\boldsymbol{\sigma}:=-\nabla u$. We begin by restoring/mimicking these three properties of the weak solution:

Definition 3.1 (Equilibrated flux reconstruction). We call an equilibrated flux reconstruction any function $\boldsymbol{\sigma}_{h}$ constructed from $u_{h}$ which satisfies

$$
\begin{gathered}
\boldsymbol{\sigma}_{h} \in \mathbf{H}(\operatorname{div}, \Omega), \\
\left(\nabla \cdot \boldsymbol{\sigma}_{h}, 1\right)_{K}=(f, 1)_{K} \quad \forall K \in \mathcal{T}_{h} .
\end{gathered}
$$

Definition 3.2 (Potential reconstruction). We call a potential reconstruction any function $s_{h}$ constructed from $u_{h}$ which satisfies

$$
s_{h} \in H_{0}^{1}(\Omega) \text {. }
$$

\subsubsection{Guaranteed reliability}

The error upper bound is straightforward:

Theorem 3.3 (A guaranteed a posteriori error estimate). Let $u$ be the weak solution of (2.2) and let $u_{h} \in H^{1}\left(\mathcal{T}_{h}\right)$ be arbitrary. Let $\boldsymbol{\sigma}_{h}$ and $s_{h}$ be respectively the equilibrated flux and potential reconstructions of Definitions 3.1 and 3.2. Then

$$
\left\|\nabla\left(u-u_{h}\right)\right\|^{2} \leq \sum_{K \in \mathcal{T}_{h}}\left(\left\|\nabla u_{h}+\boldsymbol{\sigma}_{h}\right\|_{K}+\frac{h_{K}}{\pi}\left\|f-\nabla \cdot \boldsymbol{\sigma}_{h}\right\|_{K}\right)^{2}+\sum_{K \in \mathcal{T}_{h}}\left\|\nabla\left(u_{h}-s_{h}\right)\right\|_{K}^{2} .
$$

Proof. The proof is straightforward along $[64,55,35,60,66,57,2,52,76,41,68,19,43,28,29]$. We sketch it for self-completeness. As in $[60,52]$, define $s \in H_{0}^{1}(\Omega)$ by

$$
(\nabla s, \nabla v)=\left(\nabla u_{h}, \nabla v\right) \quad \forall v \in H_{0}^{1}(\Omega) .
$$

Its existence and uniqueness follow from the Riesz representation theorem. From this projection-type construction results the Pythagorean equality

$$
\left\|\nabla\left(u-u_{h}\right)\right\|^{2}=\|\nabla(u-s)\|^{2}+\left\|\nabla\left(s-u_{h}\right)\right\|^{2}
$$

and the minimization property

$$
\left\|\nabla\left(s-u_{h}\right)\right\|^{2}=\min _{v \in H_{0}^{1}(\Omega)}\left\|\nabla\left(v-u_{h}\right)\right\|^{2} .
$$

For the first term in (3.5), using that $u-s \in H_{0}^{1}(\Omega)$, (3.4) yields

$$
\|\nabla(u-s)\|=\sup _{v \in H_{0}^{1}(\Omega) ;\|\nabla v\|=1}(\nabla(u-s), \nabla v)=\sup _{v \in H_{0}^{1}(\Omega) ;\|\nabla v\|=1}\left(\nabla\left(u-u_{h}\right), \nabla v\right) .
$$

Let $v \in H_{0}^{1}(\Omega)$ be fixed. Using $(2.2)$ and adding and subtracting $\left(\boldsymbol{\sigma}_{h}, \nabla v\right)$,

$$
\left(\nabla\left(u-u_{h}\right), \nabla v\right)=\left(f-\nabla \cdot \boldsymbol{\sigma}_{h}, v\right)-\left(\nabla u_{h}+\boldsymbol{\sigma}_{h}, \nabla v\right),
$$


where we have also employed the Green theorem $\left(\boldsymbol{\sigma}_{h}, \nabla v\right)=-\left(\nabla \cdot \boldsymbol{\sigma}_{h}, v\right)$. The Cauchy-Schwarz inequality yields

$$
-\left(\nabla u_{h}+\sigma_{h}, \nabla v\right) \leq \sum_{K \in \mathcal{T}_{h}}\left\|\nabla u_{h}+\sigma_{h}\right\|_{K}\|\nabla v\|_{K},
$$

whereas the approximate equilibrium property (3.1b), the Poincaré inequality

$$
\left\|w-\Pi_{K}^{0} w\right\|_{K} \leq C_{\mathrm{P}, K} h_{K}\|\nabla w\|_{K} \quad \forall w \in H^{1}(K),
$$

with $\Pi_{K}^{0} w$ the mean value of $w$ on $K$ and $C_{\mathrm{P}, K}=1 / \pi$ thanks to the convexity of the mesh elements $K$ (see Payne and Weinberger [63] and Bebendorf [17]), and the Cauchy-Schwarz inequality yield

$$
\left(f-\nabla \cdot \boldsymbol{\sigma}_{h}, v\right)=\sum_{K \in \mathcal{T}_{h}}\left(f-\nabla \cdot \boldsymbol{\sigma}_{h}, v-\Pi_{K}^{0} v\right)_{K} \leq \sum_{K \in \mathcal{T}_{h}} \frac{h_{K}}{\pi}\left\|f-\nabla \cdot \boldsymbol{\sigma}_{h}\right\|_{K}\|\nabla v\|_{K} .
$$

Combining these results with the Cauchy-Schwarz inequality and since any $s_{h} \in H_{0}^{1}(\Omega)$ bounds (3.6), we infer the assertion.

\subsubsection{Mixed finite element solution of Neumann problems on patches using the partition of unity}

This section describes a practical way to obtain the equilibrated flux and potential reconstructions introduced in Definitions 3.1 and 3.2. For the flux reconstruction, we rewrite equivalently the technique of [19], see also [38], proceeding as in [45]. The potential reconstruction is close to that of [29, Section 6.3]. In both cases, the equilibration goes over patches of elements $\omega_{\mathbf{a}}$ sharing a generic vertex $\mathbf{a} \in \mathcal{V}_{h}$, with $\mathbf{V}_{h}\left(\omega_{\mathbf{a}}\right) \times Q_{h}\left(\omega_{\mathbf{a}}\right)$ denoting restrictions to $\omega_{\mathbf{a}}$ of the mixed finite element spaces discussed in Section 2.4. We still only assume $u_{h} \in H^{1}\left(\mathcal{T}_{h}\right)$.

Construction 3.4 (Flux $\left.\boldsymbol{\sigma}_{h}\right)$. Let $u_{h}$ satisfy the hat-function orthogonality

$$
\left(\nabla u_{h}, \nabla \psi_{\mathbf{a}}\right)_{\omega_{\mathbf{a}}}=\left(f, \psi_{\mathbf{a}}\right)_{\omega_{\mathbf{a}}} \quad \forall \mathbf{a} \in \mathcal{V}_{h}^{\text {int }} .
$$

For each $\mathbf{a} \in \mathcal{V}_{h}$, prescribe $\boldsymbol{\varsigma}_{h}^{\mathbf{a}} \in \mathbf{V}_{h}^{\mathbf{a}}$ and $\bar{r}_{h}^{\mathbf{a}} \in Q_{h}^{\mathbf{a}}$ by solving

$$
\begin{array}{rlrl}
\left(\boldsymbol{\varsigma}_{h}^{\mathbf{a}}, \mathbf{v}_{h}\right)_{\omega_{\mathbf{a}}}-\left(\bar{r}_{h}^{\mathbf{a}}, \nabla \cdot \mathbf{v}_{h}\right)_{\omega_{\mathbf{a}}} & =-\left(\tau_{h}^{\mathbf{a}}, \mathbf{v}_{h}\right)_{\omega_{\mathbf{a}}} & & \forall \mathbf{v}_{h} \in \mathbf{V}_{h}^{\mathbf{a}}, \\
\left(\nabla \cdot \boldsymbol{\varsigma}_{h}^{\mathbf{a}}, q_{h}\right)_{\omega_{\mathbf{a}}} & =\left(g^{\mathbf{a}}, q_{h}\right)_{\omega_{\mathbf{a}}} & \forall q_{h} \in Q_{h}^{\mathbf{a}},
\end{array}
$$

with the spaces

$$
\begin{array}{lll}
\mathbf{V}_{h}^{\mathbf{a}}:=\left\{\mathbf{v}_{h} \in \mathbf{V}_{h}\left(\omega_{\mathbf{a}}\right) ; \mathbf{v}_{h} \cdot \mathbf{n}_{\omega_{\mathbf{a}}}=0 \text { on } \partial \omega_{\mathbf{a}}\right\}, & \\
Q_{h}^{\mathbf{a}}:=\left\{q_{h} \in Q_{h}\left(\omega_{\mathbf{a}}\right) ;\left(q_{h}, 1\right)_{\omega_{\mathbf{a}}}=0\right\}, & & \mathbf{a} \in \mathcal{V}_{h}^{\text {int }}, \\
\mathbf{V}_{h}^{\mathbf{a}}:=\left\{\mathbf{v}_{h} \in \mathbf{V}_{h}\left(\omega_{\mathbf{a}}\right) ; \mathbf{v}_{h} \cdot \mathbf{n}_{\omega_{\mathbf{a}}}=0 \text { on } \partial \omega_{\mathbf{a}} \backslash \partial \Omega\right\}, & \\
Q_{h}^{\mathbf{a}}:=Q_{h}\left(\omega_{\mathbf{a}}\right), & \mathbf{a} \in \mathcal{V}_{h}^{\text {ext }},
\end{array}
$$

and the right-hand sides

$$
\begin{aligned}
\tau_{h}^{\mathbf{a}} & :=\psi_{\mathbf{a}} \nabla u_{h}, \\
g^{\mathbf{a}} & :=\psi_{\mathbf{a}} f-\nabla \psi_{\mathbf{a}} \cdot \nabla u_{h} .
\end{aligned}
$$

Then, set

$$
\sigma_{h}:=\sum_{\mathbf{a} \in \mathcal{V}_{h}} \varsigma_{h}^{\mathbf{a}}
$$

In (3.11), a homogeneous Neumann (no-flux) boundary condition on the whole boundary of the patch $\omega_{\mathbf{a}}$ together with mean value zero is imposed for interior vertices, whereas the no-flux condition is only imposed in the interior of $\Omega$ for boundary vertices. Also note that by $(3.9),\left(g^{\mathbf{a}}, 1\right)_{\omega_{\mathbf{a}}}=0$ for interior vertices a, which is the Neumann compatibility condition. Existence and uniqueness of the solution to (3.10) are standard, see $[21,69,78]$. We now verify the requirements of Definition 3.1: 
Lemma 3.5 (Properties of $\left.\boldsymbol{\sigma}_{h}\right)$. Construction 3.4 yields a flux reconstruction $\boldsymbol{\sigma}_{h} \in \mathbf{H}(\operatorname{div}, \Omega)$ such that

$$
\left(f-\nabla \cdot \boldsymbol{\sigma}_{h}, v_{h}\right)_{K}=0 \quad \forall v_{h} \in Q_{h}(K) \quad \forall K \in \mathcal{T}_{h}
$$

Proof. It is clear that $\boldsymbol{\sigma}_{h} \in \mathbf{H}(\operatorname{div}, \Omega)$ as all $\boldsymbol{\varsigma}_{h}^{\mathbf{a}}$ belong to $\mathbf{H}(\operatorname{div}, \Omega)$. The facts that $\boldsymbol{\varsigma}_{h}^{\mathbf{a}} \cdot \mathbf{n}_{\omega_{\mathbf{a}}}=0$ on $\partial \omega_{\mathbf{a}}$ and $\left(g^{\mathbf{a}}, 1\right)_{\omega_{\mathbf{a}}}=0$ for $\mathbf{a} \in \mathcal{V}_{h}^{\text {int }}$ enable us to take the constants as test functions in (3.10b) and thus (3.10b) actually holds for all functions from $Q_{h}\left(\omega_{\mathbf{a}}\right)$. As the polynomials in $Q_{h}\left(\omega_{\mathbf{a}}\right)$ are discontinuous, we infer that any $q_{h} \in Q_{h}(K)$ with any $K \in \mathcal{T}_{\mathbf{a}}$ can be taken as a test function in (3.10b). Fix $K \in \mathcal{T}_{h}$ and $v_{h} \in Q_{h}(K)$. Employing that $\left.\boldsymbol{\sigma}_{h}\right|_{K}=\left.\sum_{\mathbf{a} \in \mathcal{V}_{K}} \boldsymbol{\varsigma}_{h}^{\mathbf{a}}\right|_{K}$ and (3.10b) with (3.12b),

$$
\left(f-\nabla \cdot \boldsymbol{\sigma}_{h}, v_{h}\right)_{K}=\sum_{\mathbf{a} \in \mathcal{V}_{K}}\left(\psi_{\mathbf{a}} f-\nabla \cdot \varsigma_{h}^{\mathbf{a}}, v_{h}\right)_{K}=\sum_{\mathbf{a} \in \mathcal{V}_{K}}\left(\nabla \psi_{\mathbf{a}} \cdot \nabla u_{h}, v_{h}\right)_{K}=0,
$$

where we have also used the partition of unity

$$
\left.\sum_{\mathbf{a} \in \mathcal{V}_{K}} \psi_{\mathbf{a}}\right|_{K}=\left.1\right|_{K}
$$

Remark 3.6 (Data oscillation). The orthogonality (3.14) together with the mixed finite element spaces property $\nabla \cdot \mathbf{V}_{h}(K)=Q_{h}(K)$ for any $K \in \mathcal{T}_{h}$ imply that

$$
\frac{h_{K}}{\pi}\left\|f-\nabla \cdot \sigma_{h}\right\|_{K}=\frac{h_{K}}{\pi}\left\|f-\Pi_{Q_{h}} f\right\|_{K}
$$

is actually the data oscillation term, where $\Pi_{Q_{h}}$ is the $L^{2}(\Omega)$-orthogonal projection onto $Q_{h}$. If $\left\|\nabla\left(u-u_{h}\right)\right\|$ converges as $\mathcal{O}\left(h^{p}\right), Q_{h}=\mathbb{P}_{p}\left(\mathcal{T}_{h}\right)$ or $\mathbb{Q}_{p}\left(\mathcal{T}_{h}\right)$, and $f$ is elementwise smooth, this term converges as $\mathcal{O}\left(h^{p+2}\right)$, i.e., by two orders faster.

Remark 3.7 (Local flux minimization). From (3.3), one "best" choice for the equilibrated flux reconstruc-

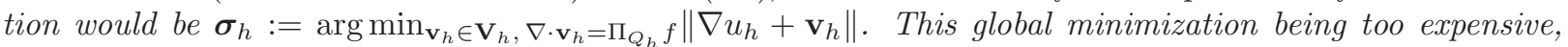
Construction 3.4 rather relies on the partition of unity by the hat functions $\psi_{\mathbf{a}}$ and finds the following local minimizers:

$$
\boldsymbol{\varsigma}_{h}^{\mathbf{a}}:=\arg \min _{\mathbf{v}_{h} \in \mathbf{V}_{h}^{\mathbf{a}}, \nabla \cdot \mathbf{v}_{h}=\Pi_{Q_{h}^{\mathbf{a}}} g^{\mathbf{a}}}\left\|\psi_{\mathbf{a}} \nabla u_{h}+\mathbf{v}_{h}\right\|_{\omega_{\mathbf{a}}} \quad \forall \mathbf{a} \in \mathcal{V}_{h}
$$

where $g^{\mathbf{a}}=\psi_{\mathbf{a}} f-\nabla \psi_{\mathbf{a}} \cdot \nabla u_{h}$ satisfies $\left.\sum_{\mathbf{a} \in \mathcal{V}_{K}} g^{\mathbf{a}}\right|_{K}=\left.f\right|_{K}$ for all $K \in \mathcal{T}_{h}$; see, e.g., [69] for the equivalence of (3.10) with (3.16).

We now turn to the potential reconstruction $s_{h}$, necessary when $u_{h} \notin H_{0}^{1}(\Omega)$ :

Construction 3.8 (Potential $s_{h}$ ). For each $\mathbf{a} \in \mathcal{V}_{h}$, prescribe $\boldsymbol{\varsigma}_{h}^{\mathbf{a}} \in \mathbf{V}_{h}^{\mathbf{a}}$ and $\bar{r}_{h}^{\mathbf{a}} \in Q_{h}^{\mathbf{a}}$ by solving

$$
\begin{array}{rlrl}
\left(\boldsymbol{\varsigma}_{h}^{\mathbf{a}}, \mathbf{v}_{h}\right)_{\omega_{\mathbf{a}}}- & \left(\bar{r}_{h}^{\mathbf{a}}, \nabla \cdot \mathbf{v}_{h}\right)_{\omega_{\mathbf{a}}}=-\left(\boldsymbol{\tau}_{h}^{\mathbf{a}}, \mathbf{v}_{h}\right)_{\omega_{\mathbf{a}}} & & \forall \mathbf{v}_{h} \in \mathbf{V}_{h}^{\mathbf{a}}, \\
\left(\nabla \cdot \boldsymbol{\varsigma}_{h}^{\mathbf{a}}, q_{h}\right)_{\omega_{\mathbf{a}}}=\left(g^{\mathbf{a}}, q_{h}\right)_{\omega_{\mathbf{a}}} & & \forall q_{h} \in Q_{h}^{\mathbf{a}},
\end{array}
$$

with the spaces

$$
\begin{aligned}
& \mathbf{V}_{h}^{\mathbf{a}}:=\left\{\mathbf{v}_{h} \in \mathbf{V}_{h}\left(\omega_{\mathbf{a}}\right) ; \mathbf{v}_{h} \cdot \mathbf{n}_{\omega_{\mathbf{a}}}=0 \text { on } \partial \omega_{\mathbf{a}}\right\} \\
& Q_{h}^{\mathbf{a}}:=\left\{q_{h} \in Q_{h}\left(\omega_{\mathbf{a}}\right) ;\left(q_{h}, 1\right)_{\omega_{\mathbf{a}}}=0\right\}
\end{aligned}
$$

and the right-hand sides

$$
\begin{aligned}
\tau_{h}^{\mathbf{a}} & :=\mathrm{R}_{\frac{\pi}{2}} \nabla\left(\psi_{\mathbf{a}} u_{h}\right), \\
g^{\mathbf{a}} & :=0 .
\end{aligned}
$$

Then, set

$$
\begin{aligned}
-\mathrm{R}_{\frac{\pi}{2}} \nabla s_{h}^{\mathbf{a}} & =\varsigma_{h}^{\mathbf{a}}, \\
s_{h}^{\mathbf{a}} & =0 \text { on } \partial \omega_{\mathbf{a}}, \\
s_{h} & :=\sum_{\mathbf{a} \in \mathcal{V}_{h}} s_{h}^{\mathbf{a}} .
\end{aligned}
$$


The local mixed finite element problem (3.17) is the same as that of Construction 3.4; only the spaces $\mathbf{V}_{h}^{\mathbf{a}}$ and $Q_{h}^{\mathbf{a}}$ differ for boundary vertices, and the right-hand sides $\tau_{h}^{\mathbf{a}}$ and $g^{\mathbf{a}}$ differ for all vertices. Existence and uniqueness of the solution to (3.17) is thus again granted. Moreover, the potential reconstruction from (3.20) is meaningful. Indeed, the fact that $\boldsymbol{\varsigma}_{h}^{\mathbf{a}} \cdot \mathbf{n}_{\omega_{\mathbf{a}}}=0$ on $\partial \omega_{\mathbf{a}}$ and $(3.19 \mathrm{~b})$ enable us to take all test functions from $Q_{h}\left(\omega_{\mathbf{a}}\right)$ in $(3.17 \mathrm{~b})$. Thus $\nabla \cdot \boldsymbol{\varsigma}_{h}^{\mathbf{a}}=0$ on each $K \in \mathcal{T}_{\mathbf{a}}$ and, consequently, there exists a piecewise polynomial $s_{h}^{\mathbf{a}}$ satisfying (3.20a) on each $K \in \mathcal{T}_{\mathbf{a}}$. The continuity of the normal trace of $\varsigma_{h}^{\mathbf{a}}$ over the interior edges of $\mathcal{T}_{\mathbf{a}}$ then implies the continuity of the tangential trace of $s_{h}^{\mathbf{a}}$ over the interior edges of $\mathcal{T}_{\mathbf{a}}$. Finally, the normal trace of $\boldsymbol{\varsigma}_{h}^{\mathbf{a}}$ being zero on $\partial \omega_{\mathbf{a}}, s_{h}^{\mathbf{a}}$ is constant on $\partial \omega_{\mathbf{a}}$ and we can fix it to zero on $\partial \omega_{\mathbf{a}}$ by $(3.20 \mathrm{~b})$. Hence, $s_{h}^{\mathbf{a}}$ is uniquely defined. This function is a piecewise polynomial in $H_{0}^{1}\left(\omega_{\mathbf{a}}\right)$ for all $\mathbf{a} \in \mathcal{V}_{h}$. Altogether, we have:

Lemma 3.9 (Properties of $s_{h}$ ). Construction 3.8 yields a potential reconstruction $s_{h} \in H_{0}^{1}(\Omega)$, i.e., (3.2) holds.

Remark 3.10 (Local potential minimization). From (3.3), one "best" choice for the potential reconstruction would be $s_{h}:=\arg \min _{v_{h} \in V_{h}}\left\|\nabla\left(u_{h}-v_{h}\right)\right\|$ for some finite-dimensional subspace $V_{h}$ of $H_{0}^{1}(\Omega)$. This global minimization being too expensive, we observe, as in Remark 3.7, that Construction 3.8 rather relies on to the following partition-of-unity-based local minimization:

$$
\boldsymbol{\varsigma}_{h}^{\mathbf{a}}:=\arg \min _{\mathbf{v}_{h} \in \mathbf{V}_{h}^{\mathbf{a}}, \nabla \cdot \mathbf{v}_{h}=0}\left\|\mathrm{R}_{\frac{\pi}{2}} \nabla\left(\psi_{\mathbf{a}} u_{h}\right)+\mathbf{v}_{h}\right\|_{\omega_{\mathbf{a}}} \quad \forall \mathbf{a} \in \mathcal{V}_{h}
$$

Now, the divergence-free $R T$ functions from $\mathbf{V}_{h}^{\mathbf{a}}$ of degree $p$ on triangles are rotated gradients of polynomials from $\mathbb{P}_{p+1}\left(\mathcal{T}_{\mathbf{a}}\right) \cap H_{0}^{1}\left(\omega_{\mathbf{a}}\right)$, see [21, Corollary 3.2]. Similarly, divergence-free RT functions from $\mathbf{V}_{h}^{\mathbf{a}}$ of degree $p$ on rectangles are rotated gradients of polynomials from $\mathbb{Q}_{p+1}\left(\mathcal{T}_{\mathbf{a}}\right) \cap H_{0}^{1}\left(\omega_{\mathbf{a}}\right)$, see [21, Lemma 3.3]. Denote the resulting spaces of scalar piecewise polynomials $V_{h}^{\mathbf{a}}$. Then, (3.21) together with (3.20a)-(3.20b) can be further equivalently rewritten as

$$
s_{h}^{\mathbf{a}}:=\arg \min _{v_{h} \in V_{h}^{\mathbf{a}}}\left\|\nabla\left(\psi_{\mathbf{a}} u_{h}-v_{h}\right)\right\|_{\omega_{\mathbf{a}}} \quad \forall \mathbf{a} \in \mathcal{V}_{h},
$$

which is further equivalent to finding $s_{h}^{\mathbf{a}} \in V_{h}^{\mathbf{a}}$ such that

$$
\left(\nabla s_{h}^{\mathbf{a}}, \nabla v_{h}\right)=\left(\nabla\left(\psi_{\mathbf{a}} u_{h}\right), \nabla v_{h}\right) \quad \forall v_{h} \in V_{h}^{\mathbf{a}},
$$

showing that $s_{h}^{\mathbf{a}}$ can also be computed by solving a discrete problem in primal form.

Remark 3.11 (Alternative potential reconstruction). An alternative potential reconstruction, close to that of [29, Section 6.3] is possible under the assumption

$$
\left(\nabla u_{h}, \mathrm{R}_{\frac{\pi}{2}} \nabla \psi_{\mathbf{a}}\right)_{\omega_{\mathbf{a}}}=0 \quad \forall \mathbf{a} \in \mathcal{V}_{h}
$$

Set

$$
\begin{aligned}
\tau_{h}^{\mathbf{a}} & :=\psi_{\mathbf{a}} \mathrm{R}_{\frac{\pi}{2}} \nabla u_{h}, \\
g^{\mathbf{a}} & :=\left(\mathrm{R}_{\frac{\pi}{2}} \nabla \psi_{\mathbf{a}}\right) \cdot \nabla u_{h},
\end{aligned}
$$

and use (3.17)-(3.18) together with $\boldsymbol{\varsigma}_{h}:=\sum_{\mathbf{a} \in \mathcal{V}_{h}} \boldsymbol{\varsigma}_{h}^{\mathbf{a}}$. This yields $\boldsymbol{\varsigma}_{h} \in \mathbf{V}_{h}$ such that $\boldsymbol{\varsigma}_{h} \cdot \mathbf{n}_{\Omega}=0$ on $\partial \Omega$. Moreover, proceeding as in Lemma 3.5, one readily checks that $\nabla \cdot \varsigma_{h}=0$. Thus, there exists a piecewise polynomial $s_{h}$ in $H_{0}^{1}(\Omega)$ such that $-\mathrm{R}_{\frac{\pi}{2}} \nabla s_{h}=\varsigma_{h}$. In contrast to [29, Section 6.3], but similarly to [29, Section 6.5], $g^{\mathbf{a}}$ is nonzero, as it is given by (3.23b), and this turns out to be essential for the local efficiency in Section 3.2. The advantage of Construction 3.8 is that condition (3.22) is not needed. The advantage of Construction (3.22)-(3.23) is that the local efficiency is proven with a simpler constant; see Remark 3.14 below.

\subsection{Polynomial-degree-robust efficiency}

We show here that, under the assumption of shape-regular meshes, the a posteriori error estimate of Theorem 3.3, with the Constructions 3.4 and 3.8, is also a lower bound for the error $\left\|\nabla\left(u-u_{h}\right)\right\|$, up to a generic constant only depending on the shape-regularity parameter $\kappa_{\mathcal{T}}$. We proceed in three steps. In Section 3.2.1, 
we introduce primal continuous problems on patches such that the energy norms of their solutions represent lower bounds of the local errors in the patches. In Section 3.2.2, we show that the local constructions of Section 3.1.3 represent lower bounds, up to a polynomial-degree-independent constant, of the energy norms of the continuous solutions from Section 3.2.1. Finally, in Section 3.2.3, elementwise local lower bounds for the actual estimators are derived from the results of Section 3.2.1 and Section 3.2.2.

\subsubsection{Continuous-level problems with hat functions on patches}

The following result has been shown in $[24,18]$ :

Lemma 3.12 (Continuous efficiency, flux reconstruction). Let $u$ be the weak solution of (2.2) and let $u_{h} \in H^{1}\left(\mathcal{T}_{h}\right)$ be arbitrary. Let $\mathbf{a} \in \mathcal{V}_{h}$ and let $r_{\mathbf{a}} \in H_{*}^{1}\left(\omega_{\mathbf{a}}\right)$ solve

$$
\left(\nabla r_{\mathbf{a}}, \nabla v\right)_{\omega_{\mathbf{a}}}=-\left(\boldsymbol{\tau}_{h}^{\mathbf{a}}, \nabla v\right)_{\omega_{\mathbf{a}}}+\left(g^{\mathbf{a}}, v\right)_{\omega_{\mathbf{a}}} \quad \forall v \in H_{*}^{1}\left(\omega_{\mathbf{a}}\right)
$$

with the space

$$
\begin{array}{ll}
H_{*}^{1}\left(\omega_{\mathbf{a}}\right):=\left\{v \in H^{1}\left(\omega_{\mathbf{a}}\right) ;(v, 1)_{\omega_{\mathbf{a}}}=0\right\}, & \mathbf{a} \in \mathcal{V}_{h}^{\text {int }}, \\
H_{*}^{1}\left(\omega_{\mathbf{a}}\right):=\left\{v \in H^{1}\left(\omega_{\mathbf{a}}\right) ; v=0 \text { on } \partial \omega_{\mathbf{a}} \cap \partial \Omega\right\}, & \mathbf{a} \in \mathcal{V}_{h}^{\text {ext }},
\end{array}
$$

and the right-hand sides $\boldsymbol{\tau}_{h}^{\mathbf{a}}$ and $g^{\mathbf{a}}$ from Construction 3.4. Then there exists a constant $C_{\mathrm{cont}, \mathrm{PF}}>0$ only depending on the shape-regularity parameter $\kappa_{\mathcal{T}}$ such that

$$
\left\|\nabla r_{\mathbf{a}}\right\|_{\omega_{\mathbf{a}}} \leq C_{\text {cont }, \mathrm{PF}}\left\|\nabla\left(u-u_{h}\right)\right\|_{\omega_{\mathbf{a}}}
$$

Proof. We include the proof for insight and later use. There holds

$$
\left\|\nabla r_{\mathbf{a}}\right\|_{\omega_{\mathbf{a}}}=\sup _{v \in H_{*}^{1}\left(\omega_{\mathbf{a}}\right) ;\|\nabla v\|_{\omega_{\mathbf{a}}}=1}\left(\nabla r_{\mathbf{a}}, \nabla v\right)_{\omega_{\mathbf{a}}} .
$$

Fix $v \in H_{*}^{1}\left(\omega_{\mathbf{a}}\right)$ with $\|\nabla v\|_{\omega_{\mathbf{a}}}=1$. Definitions (3.24) and (3.12), the fact that $\psi_{\mathbf{a}} v \in H_{0}^{1}\left(\omega_{\mathbf{a}}\right)$, the characterization (2.2) of the weak solution, and the Cauchy-Schwarz inequality imply

$$
\begin{aligned}
\left(\nabla r_{\mathbf{a}}, \nabla v\right)_{\omega_{\mathbf{a}}} & =-\left(\psi_{\mathbf{a}} \nabla u_{h}, \nabla v\right)_{\omega_{\mathbf{a}}}+\left(\psi_{\mathbf{a}} f-\nabla \psi_{\mathbf{a}} \cdot \nabla u_{h}, v\right)_{\omega_{\mathbf{a}}} \\
& =\left(f, \psi_{\mathbf{a}} v\right)_{\omega_{\mathbf{a}}}-\left(\nabla u_{h}, \nabla\left(\psi_{\mathbf{a}} v\right)\right)_{\omega_{\mathbf{a}}} \\
& =\left(\nabla\left(u-u_{h}\right), \nabla\left(\psi_{\mathbf{a}} v\right)\right)_{\omega_{\mathbf{a}}} \\
& \leq\left\|\nabla\left(u-u_{h}\right)\right\|_{\omega_{\mathbf{a}}}\left\|\nabla\left(\psi_{\mathbf{a}} v\right)\right\|_{\omega_{\mathbf{a}}}
\end{aligned}
$$

Next,

$$
\begin{aligned}
\left\|\nabla\left(\psi_{\mathbf{a}} v\right)\right\|_{\omega_{\mathbf{a}}} & =\left\|\nabla \psi_{\mathbf{a}} v+\psi_{\mathbf{a}} \nabla v\right\|_{\omega_{\mathbf{a}}} \\
& \leq\left\|\nabla \psi_{\mathbf{a}}\right\|_{\infty, \omega_{\mathbf{a}}}\|v\|_{\omega_{\mathbf{a}}}+\left\|\psi_{\mathbf{a}}\right\|_{\infty, \omega_{\mathbf{a}}}\|\nabla v\|_{\omega_{\mathbf{a}}} \\
& \leq 1+C_{\mathrm{PF}, \omega_{\mathbf{a}}} h_{\omega_{\mathbf{a}}}\left\|\nabla \psi_{\mathbf{a}}\right\|_{\infty, \omega_{\mathbf{a}}}
\end{aligned}
$$

employing $\|\nabla v\|_{\omega_{\mathbf{a}}}=1,\left\|\psi_{\mathbf{a}}\right\|_{\infty, \omega_{\mathbf{a}}}=1$, the Poincaré inequality (3.7) on the patch $\omega_{\mathbf{a}}$ for $\mathbf{a} \in \mathcal{V}_{h}^{\text {int }}$, the Friedrichs inequality

$$
\|w\|_{\omega_{\mathbf{a}}} \leq C_{\mathrm{F}, \omega_{\mathbf{a}}} h_{\omega_{\mathbf{a}}}\|\nabla w\|_{\omega_{\mathbf{a}}} \quad \forall w \in H_{*}^{1}\left(\omega_{\mathbf{a}}\right)
$$

for $\mathbf{a} \in \mathcal{V}_{h}^{\text {ext }}$, and setting $C_{\mathrm{PF}, \omega_{\mathbf{a}}}:=C_{\mathrm{P}, \omega_{\mathbf{a}}}$ if $\mathbf{a} \in \mathcal{V}_{h}^{\text {int }}$ and $C_{\mathrm{PF}, \omega_{\mathbf{a}}}:=C_{\mathrm{F}, \omega_{\mathbf{a}}}$ if $\mathbf{a} \in \mathcal{V}_{h}^{\text {ext }}$. For the values of constants in (3.7) and (3.30), in particular on nonconvex patches $\omega_{\mathbf{a}}$, we refer to Eymard et al. [47, 48], Carstensen and Funken [25], Veeser and Verfürth [73], Šebestová and Vejchodský [70], as well as to the references therein. Thus (3.26) follows with $C_{\text {cont,PF }}:=\max _{\mathbf{a} \in \mathcal{V}_{h}}\left\{1+C_{\mathrm{PF}, \omega_{\mathbf{a}}} h_{\omega_{\mathbf{a}}}\left\|\nabla \psi_{\mathbf{a}}\right\|_{\infty, \omega_{\mathbf{a}}}\right\}$.

A related but different problem (namely on the right-hand side) to (3.24) appears in [29, Section 6.5]. We now prove a new crucial estimate for its solution. The proof hinges on the additional assumption of the continuity in mean of the jumps of the approximate solution $u_{h}$ (note that this assumption implies (3.22)). We refer to Section 4.3.2 for a more refined analysis when this assumption is not met. 
Lemma 3.13 (Continuous efficiency, potential reconstruction). Let $u$ be the weak solution of (2.2) and let $u_{h} \in H^{1}\left(\mathcal{T}_{h}\right)$ satisfy

$$
\left\langle\llbracket u_{h} \rrbracket, 1\right\rangle_{e}=0 \quad \forall e \in \mathcal{E}_{h} .
$$

Let $\mathbf{a} \in \mathcal{V}_{h}$ and let $r_{\mathbf{a}} \in H_{*}^{1}\left(\omega_{\mathbf{a}}\right)$ solve

$$
\left(\nabla r_{\mathbf{a}}, \nabla v\right)_{\omega_{\mathbf{a}}}=-\left(\boldsymbol{\tau}_{h}^{\mathbf{a}}, \nabla v\right)_{\omega_{\mathbf{a}}}+\left(g^{\mathbf{a}}, v\right)_{\omega_{\mathbf{a}}} \quad \forall v \in H_{*}^{1}\left(\omega_{\mathbf{a}}\right)
$$

with the space

$$
H_{*}^{1}\left(\omega_{\mathbf{a}}\right):=\left\{v \in H^{1}\left(\omega_{\mathbf{a}}\right) ;(v, 1)_{\omega_{\mathbf{a}}}=0\right\}
$$

and the right-hand sides $\tau_{h}^{\mathbf{a}}$ and $g^{\mathbf{a}}$ from Construction 3.8. Then there exists a constant $C_{\mathrm{cont}, \mathrm{bPF}}>0$ only depending on the shape-regularity parameter $\kappa_{\mathcal{T}}$ such that

$$
\left\|\nabla r_{\mathbf{a}}\right\|_{\omega_{\mathbf{a}}} \leq C_{\text {cont }, \mathrm{bPF}}\left\|\nabla\left(u-u_{h}\right)\right\|_{\omega_{\mathbf{a}}} .
$$

Proof. We start again from (3.27) and fix $v \in H_{*}^{1}\left(\omega_{\mathbf{a}}\right)$ with $\|\nabla v\|_{\omega_{\mathbf{a}}}=1$. For an arbitrary $\tilde{u} \in H^{1}\left(\omega_{\mathbf{a}}\right)$ such that $(\tilde{u}, 1)_{\omega_{\mathbf{a}}}=\left(u_{h}, 1\right)_{\omega_{\mathbf{a}}}$ if $\mathbf{a} \in \mathcal{V}_{h}^{\text {int }}$ and $\tilde{u}=0$ on $\partial \omega_{\mathbf{a}} \cap \partial \Omega$ if $\mathbf{a} \in \mathcal{V}_{h}^{\text {ext }}$, we observe that

$$
\left(\mathrm{R}_{\frac{\pi}{2}} \nabla\left(\psi_{\mathbf{a}} \tilde{u}\right), \nabla v\right)_{\omega_{\mathbf{a}}}=0 .
$$

Thus, using (3.32) with (3.19) and the Cauchy-Schwarz inequality, we arrive at

$$
\begin{aligned}
\left(\nabla r_{\mathbf{a}}, \nabla v\right)_{\omega_{\mathbf{a}}} & =-\left(\mathrm{R}_{\frac{\pi}{2}} \nabla\left(\psi_{\mathbf{a}} u_{h}\right), \nabla v\right)_{\omega_{\mathbf{a}}}=\left(\mathrm{R}_{\frac{\pi}{2}} \nabla\left(\psi_{\mathbf{a}}\left(\tilde{u}-u_{h}\right)\right), \nabla v\right)_{\omega_{\mathbf{a}}} \\
& \leq\left\|\mathrm{R}_{\frac{\pi}{2}} \nabla\left(\psi_{\mathbf{a}}\left(\tilde{u}-u_{h}\right)\right)\right\|_{\omega_{\mathbf{a}}}\|\nabla v\|_{\omega_{\mathbf{a}}}=\left\|\nabla\left(\psi_{\mathbf{a}}\left(\tilde{u}-u_{h}\right)\right)\right\|_{\omega_{\mathbf{a}}} .
\end{aligned}
$$

We next intend to proceed as in (3.29), with $\tilde{u}-u_{h}$ in place of $v$. The difference is that now $\tilde{u}-u_{h}$ does not belong to $H^{1}\left(\omega_{\mathbf{a}}\right)$, with zero trace on $\partial \omega_{\mathbf{a}} \cap \partial \Omega$ for $\mathbf{a} \in \mathcal{V}_{h}^{\text {ext }}$, but is a piecewise $H^{1}$ function from $H^{1}\left(\mathcal{T}_{\mathbf{a}}\right)$. There is, fortunately, the continuity in mean of the jumps owing to assumption (3.31), and in particular $\left\langle\tilde{u}-u_{h}, 1\right\rangle_{e}=0$ for all edges $e$ located in $\partial \omega_{\mathbf{a}} \cap \partial \Omega$ for $\mathbf{a} \in \mathcal{V}_{h}^{\text {ext }}$, as well as $\left(\tilde{u}-u_{h}, 1\right)_{\omega_{\mathbf{a}}}=0$ for $\mathbf{a} \in \mathcal{V}_{h}^{\text {int }}$. Thus the Poincaré inequality (3.7) (on the patch $\omega_{\mathbf{a}}$ ) and the Friedrichs inequality (3.30) have to be replaced by their broken versions (see Brenner [20] or [75] and the references therein), leading to

$$
\left\|\nabla\left(\psi_{\mathbf{a}}\left(\tilde{u}-u_{h}\right)\right)\right\|_{\omega_{\mathbf{a}}} \leq\left(1+C_{\mathrm{bPF}, \omega_{\mathbf{a}}} h_{\omega_{\mathbf{a}}}\left\|\nabla \psi_{\mathbf{a}}\right\|_{\infty, \omega_{\mathbf{a}}}\right)\left\|\nabla\left(\tilde{u}-u_{h}\right)\right\|_{\omega_{\mathbf{a}}}
$$

Now it suffices to choose for $\tilde{u}$ the weak solution $u$ shifted on interior patches by a constant such that $\left(\tilde{u}-u_{h}, 1\right)_{\omega_{\mathbf{a}}}=0$ to infer $(3.34)$ with $C_{\mathrm{cont}, \mathrm{bPF}}:=\max _{\mathbf{a} \in \mathcal{V}_{h}}\left\{1+C_{\mathrm{bPF}, \omega_{\mathbf{a}}} h_{\omega_{\mathbf{a}}}\left\|\nabla \psi_{\mathbf{a}}\right\|_{\infty, \omega_{\mathbf{a}}}\right\}$.

Remark 3.14 (Efficiency for the potential reconstruction of Remark 3.11). Efficiency for the potential reconstruction of Remark 3.11 can be shown as above. In particular, problem (3.32) with the right-hand sides $\tau_{h}^{\mathbf{a}}$ and $g^{\mathbf{a}}$ from Remark 3.11 and $H_{*}^{1}\left(\omega_{\mathbf{a}}\right)$ still defined by (3.33) reads (cf. [29, Section 6.5]): find $r_{\mathbf{a}} \in H_{*}^{1}\left(\omega_{\mathbf{a}}\right)$ such that

$$
\left(\nabla r_{\mathbf{a}}, \nabla v\right)_{\omega_{\mathbf{a}}}=\left(\nabla u_{h}, \mathrm{R}_{\frac{\pi}{2}} \nabla\left(\psi_{\mathbf{a}} v\right)\right)_{\omega_{\mathbf{a}}} \quad \forall v \in H_{*}^{1}\left(\omega_{\mathbf{a}}\right)
$$

An essential property is that $\left(\nabla u, \mathrm{R}_{\frac{\pi}{2}} \nabla\left(\psi_{\mathbf{a}} v\right)\right)_{\omega_{\mathbf{a}}}=0$. Thus,

$$
\left(\nabla r_{\mathbf{a}}, \nabla v\right)_{\omega_{\mathbf{a}}} \leq\left\|\nabla\left(u-u_{h}\right)\right\|_{\omega_{\mathbf{a}}}\left\|\mathrm{R}_{\frac{\pi}{2}} \nabla\left(\psi_{\mathbf{a}} v\right)\right\|_{\omega_{\mathbf{a}}}=\left\|\nabla\left(u-u_{h}\right)\right\|_{\omega_{\mathbf{a}}}\left\|\nabla\left(\psi_{\mathbf{a}} v\right)\right\|_{\omega_{\mathbf{a}}}
$$

for any $v \in H_{*}^{1}\left(\omega_{\mathbf{a}}\right)$, and we conclude by (3.29) that

$$
\left\|\nabla r_{\mathbf{a}}\right\|_{\omega_{\mathbf{a}}} \leq C_{\text {cont }, \mathrm{P}}\left\|\nabla\left(u-u_{h}\right)\right\|_{\omega_{\mathbf{a}}}
$$

holds in this case, with $C_{\mathrm{cont}, \mathrm{P}}:=\max _{\mathbf{a} \in \mathcal{V}_{h}}\left\{1+C_{\mathrm{P}, \omega_{\mathbf{a}}} h_{\omega_{\mathbf{a}}}\left\|\nabla \psi_{\mathbf{a}}\right\|_{\infty, \omega_{\mathbf{a}}}\right\}$, thereby requiring only the Poincaré inequality. 
Remark 3.15 (Dual and dual mixed formulations). For a vertex $\mathbf{a} \in \mathcal{V}_{h}$, consider the following dual formulation: Find $\varsigma_{\mathbf{a}} \in \mathbf{H}_{*}\left(\operatorname{div}, \omega_{\mathbf{a}}\right)$ with $\nabla \cdot \varsigma_{\mathbf{a}}=g^{\mathbf{a}}$ such that

$$
\left(\boldsymbol{\varsigma}_{\mathbf{a}}, \mathbf{v}\right)_{\omega_{\mathbf{a}}}=-\left(\boldsymbol{\tau}_{h}^{\mathbf{a}}, \mathbf{v}\right)_{\omega_{\mathbf{a}}} \quad \forall \mathbf{v} \in \mathbf{H}_{*}\left(\operatorname{div}, \omega_{\mathbf{a}}\right) \text { with } \nabla \cdot \mathbf{v}=0 .
$$

Here, $\mathbf{H}_{*}\left(\operatorname{div}, \omega_{\mathbf{a}}\right)$ stands for $\mathbf{H}\left(\operatorname{div}, \omega_{\mathbf{a}}\right)$ functions with zero normal trace in the appropriate sense on $\partial \omega_{\mathbf{a}}$ for $\mathbf{a} \in \mathcal{V}_{h}^{\text {int }}$ and for $\mathbf{H}\left(\operatorname{div}, \omega_{\mathbf{a}}\right)$ functions with zero normal trace in the appropriate sense on $\partial \omega_{\mathbf{a}} \backslash \partial \Omega$ for $\mathbf{a} \in \mathcal{V}_{h}^{\text {ext }}$. Similarly, consider the dual mixed formulation: Find $\boldsymbol{\varsigma}_{\mathbf{a}} \in \mathbf{H}_{*}\left(\operatorname{div}, \omega_{\mathbf{a}}\right)$ and $\bar{r}^{\mathbf{a}} \in L_{*}^{2}\left(\omega_{\mathbf{a}}\right)$ such that

$$
\begin{aligned}
\left(\boldsymbol{\varsigma}_{\mathbf{a}}, \mathbf{v}\right)_{\omega_{\mathbf{a}}}- & \left(\bar{r}^{\mathbf{a}}, \nabla \cdot \mathbf{v}\right)_{\omega_{\mathbf{a}}}=-\left(\boldsymbol{\tau}_{h}^{\mathbf{a}}, \mathbf{v}\right)_{\omega_{\mathbf{a}}} & & \forall \mathbf{v} \in \mathbf{H}_{*}\left(\operatorname{div}, \omega_{\mathbf{a}}\right), \\
\left(\nabla \cdot \boldsymbol{\varsigma}_{\mathbf{a}}, q\right)_{\omega_{\mathbf{a}}} & =\left(g^{\mathbf{a}}, q\right)_{\omega_{\mathbf{a}}} & & \forall q \in L_{*}^{2}\left(\omega_{\mathbf{a}}\right) .
\end{aligned}
$$

Here, $L_{*}^{2}\left(\omega_{\mathbf{a}}\right)$ is the space of functions from $L^{2}\left(\omega_{\mathbf{a}}\right)$ with zero mean value for $\mathbf{a} \in \mathcal{V}_{h}^{\text {int }}$ and $L^{2}\left(\omega_{\mathbf{a}}\right)$ for $\mathbf{a} \in \mathcal{V}_{h}^{\text {ext }}$. It is classical that the problems (3.37) and (3.38) are equivalent to the primal formulation (3.24), with $\boldsymbol{\varsigma}_{\mathbf{a}}=-\nabla r_{\mathbf{a}}-\boldsymbol{\tau}_{h}^{\mathbf{a}}$. Then, (3.10) is the natural finite element discretization of (3.38). The same links hold true in the potential reconstruction cases.

\subsubsection{Uniform-in-polynomial-degree stability of mixed finite element methods}

The following crucial result has been shown in [18, Theorem 7], based on [34, Corollary 3.4] and [36, Theorem 7.1] for triangles and on [18, Theorem 7] and [33] on rectangles:

Corollary 3.16 (Uniform stability of mixed finite element methods). Let $\mathbf{a} \in \mathcal{V}_{h}$ and let $\tau_{h}^{\mathbf{a}}$ and $g^{\mathbf{a}}$ result from either Construction 3.4 or Construction 3.8. Suppose that

$$
\begin{aligned}
\left.\tau_{h}^{\mathbf{a}}\right|_{K} \in \mathbf{V}_{h}(K) & \forall K \in \mathcal{T}_{\mathbf{a}}, \\
\left.g^{\mathbf{a}}\right|_{K} \in Q_{h}(K) & \forall K \in \mathcal{T}_{\mathbf{a}} .
\end{aligned}
$$

Let $r_{\mathbf{a}} \in H_{*}^{1}\left(\omega_{\mathbf{a}}\right)$ accordingly solve either (3.24) with $H_{*}^{1}\left(\omega_{\mathbf{a}}\right)$ given by (3.25) or (3.32) with $H_{*}^{1}\left(\omega_{\mathbf{a}}\right)$ given by (3.33). Let finally $\boldsymbol{\varsigma}_{h}^{\text {a }}$ be the solution of either (3.10) or (3.17). Then there exists a constant $C_{\mathrm{st}}>0$ only depending on the shape-regularity parameter $\kappa_{\mathcal{T}}$ such that

$$
\left\|\boldsymbol{s}_{h}^{\mathbf{a}}+\tau_{h}^{\mathbf{a}}\right\|_{\omega_{\mathbf{a}}} \leq C_{\mathrm{st}}\left\|\nabla r_{\mathbf{a}}\right\|_{\omega_{\mathbf{a}}}
$$

Proof. We have from (3.24) or (3.32), using (3.27),

$$
\begin{aligned}
\left\|\nabla r_{\mathbf{a}}\right\|_{\omega_{\mathbf{a}}} & =\sup _{v \in H_{*}^{1}\left(\omega_{\mathbf{a}}\right) ;\|\nabla v\|_{\omega_{\mathbf{a}}}=1}\left\{-\left(\boldsymbol{\tau}_{h}^{\mathbf{a}}, \nabla v\right)_{\omega_{\mathbf{a}}}+\left(g^{\mathbf{a}}, v\right)_{\omega_{\mathbf{a}}}\right\} \\
& =\sup _{v \in H_{*}^{1}\left(\omega_{\mathbf{a}}\right) ;\|\nabla v\|_{\omega_{\mathbf{a}}}=1}\left\{\sum_{e \in \mathcal{E}_{h}, \mathbf{a} \in e}\langle\underbrace{\llbracket-\tau_{h}^{\mathbf{a}} \cdot \mathbf{n}_{e} \rrbracket}_{r_{e}}, v\rangle_{e}+\sum_{K \in \mathcal{T}_{\mathbf{a}}}(\underbrace{\nabla \cdot \tau_{h}^{\mathbf{a}}+g^{\mathbf{a}}}_{r_{K}}, v)_{K}\right\},
\end{aligned}
$$

so that $\left\|\nabla r_{\mathbf{a}}\right\|_{\omega_{\mathbf{a}}}$ in our notation is $\|r\|_{\left[H^{1}(\omega) / \mathbb{R}\right]^{*}}$ in the notation of [18]. Simultaneously, (3.16) and (3.21) read $\left\|\boldsymbol{s}_{h}^{\mathbf{a}}+\boldsymbol{\tau}_{h}^{\mathbf{a}}\right\|_{\omega_{\mathbf{a}}}=\inf _{\mathbf{v}_{h} \in \mathbf{V}_{h}^{\mathbf{a}}, \nabla \cdot \mathbf{v}_{h}=g^{\mathbf{a}}}\left\|\mathbf{v}_{h}+\boldsymbol{\tau}_{h}^{\mathbf{a}}\right\|_{\omega_{\mathbf{a}}}$. Setting $\boldsymbol{\delta}_{h}^{\mathbf{a}}:=\boldsymbol{\varsigma}_{h}^{\mathbf{a}}+\boldsymbol{\tau}_{h}^{\mathbf{a}}$, we see that

$$
\left\|\boldsymbol{s}_{h}^{\mathbf{a}}+\tau_{h}^{\mathbf{a}}\right\|_{\omega_{\mathbf{a}}}=\left\|\boldsymbol{\delta}_{h}^{\mathbf{a}}\right\|_{\omega_{\mathbf{a}}}=\inf _{\mathbf{v}_{h} \in \mathbf{V}_{h}^{\mathbf{a}}\left(\mathcal{T}_{\mathbf{a}}\right),\left.\nabla \cdot \mathbf{v}_{h}\right|_{K}=\left.\left(\nabla \cdot \mathcal{T}_{h}^{\mathbf{a}}+g^{\mathbf{a}}\right)\right|_{K} \forall K \in \mathcal{T}_{\mathbf{a}}}\left\|\mathbf{v}_{h}\right\|_{\omega_{\mathbf{a}}},
$$

where $\mathbf{V}_{h}^{\mathbf{a}}\left(\mathcal{T}_{\mathbf{a}}\right)$ is the broken version of $\mathbf{V}_{h}^{\mathbf{a}}$ with normal jumps imposed by $\llbracket \tau_{h}^{\mathbf{a}} \cdot \mathbf{n}_{e} \rrbracket$, which is the form employed in [18, Theorem 7].

\subsubsection{Polynomial-degree-robust efficiency}

We are now ready to prove the main result of this paper: 
Theorem 3.17 (Polynomial-degree-robust efficiency). Let $u$ be the weak solution of (2.2). Let $u_{h}$ be a piecewise polynomial and consider Construction 3.4 of $\boldsymbol{\sigma}_{h}$ with the spaces $\mathbf{V}_{h}$ and $Q_{h}$ satisfying, for all $\mathbf{a} \in \mathcal{V}_{h}$,

$$
\begin{aligned}
\left.\left(\psi_{\mathbf{a}} \nabla u_{h}\right)\right|_{K} \in \mathbf{V}_{h}(K) & \forall K \in \mathcal{T}_{\mathbf{a}}, \\
\left.\left(\nabla \psi_{\mathbf{a}} \cdot \nabla u_{h}\right)\right|_{K} \in Q_{h}(K) & \forall K \in \mathcal{T}_{\mathbf{a}} .
\end{aligned}
$$

Then,

$$
\begin{aligned}
\left\|\nabla u_{h}+\boldsymbol{\sigma}_{h}\right\|_{K} \leq & C_{\text {st }} C_{\text {cont }, \text { PF }} \sum_{\mathbf{a} \in \mathcal{V}_{K}}\left\|\nabla\left(u-u_{h}\right)\right\|_{\omega_{\mathbf{a}}} \\
& +C_{\text {st }} \sum_{\mathbf{a} \in \mathcal{V}_{K}}\left\{\sum_{K^{\prime} \in \mathcal{T}_{\mathbf{a}}}\left(\frac{h_{K^{\prime}}}{\pi}\left\|\psi_{\mathbf{a}} f-\Pi_{Q_{h}}\left(\psi_{\mathbf{a}} f\right)\right\|_{K^{\prime}}\right)^{2}\right\}^{1 / 2},
\end{aligned}
$$

for all $K \in \mathcal{T}_{h}$, with the constants $C_{\mathrm{st}}$ of (3.40) and $C_{\mathrm{cont}, \mathrm{PF}}$ of (3.26), respectively. Consider now Construction 3.8 of $s_{h}$ with the space $\mathbf{V}_{h}$ satisfying, for all $\mathbf{a} \in \mathcal{V}_{h}$,

$$
\left.\left(\mathrm{R}_{\frac{\pi}{2}} \nabla\left(\psi_{\mathbf{a}} u_{h}\right)\right)\right|_{K} \in \mathbf{V}_{h}(K) \quad \forall K \in \mathcal{T}_{\mathbf{a}} .
$$

Assume in addition that $u_{h}$ verifies the zero-mean condition (3.31). Then,

$$
\left\|\nabla\left(u_{h}-s_{h}\right)\right\|_{K} \leq C_{\mathrm{st}} C_{\mathrm{cont}, \mathrm{bPF}} \sum_{\mathbf{a} \in \mathcal{V}_{K}}\left\|\nabla\left(u-u_{h}\right)\right\|_{\omega_{\mathbf{a}}}
$$

for all $K \in \mathcal{T}_{h}$, with the constants $C_{\text {st }}$ of (3.40) and $C_{\text {cont,bPF }}$ of (3.34), respectively.

Proof. (1) We first prove (3.44). Let $K \in \mathcal{T}_{h}$. Using Construction 3.8, the decomposition $\left.s_{h}\right|_{K}=$ $\left.\sum_{\mathbf{a} \in \mathcal{V}_{K}} s_{h}^{\mathbf{a}}\right|_{K}$, the partition of unity (3.15), and the triangle inequality, we infer that

$$
\begin{aligned}
\left\|\nabla\left(u_{h}-s_{h}\right)\right\|_{K} & =\left\|\left.\sum_{\mathbf{a} \in \mathcal{V}_{K}}\left(\nabla\left(\psi_{\mathbf{a}} u_{h}-s_{h}^{\mathbf{a}}\right)\right)\right|_{K}\right\|_{K} \leq \sum_{\mathbf{a} \in \mathcal{V}_{K}}\left\|\nabla\left(\psi_{\mathbf{a}} u_{h}-s_{h}^{\mathbf{a}}\right)\right\|_{K} \\
& \left.=\sum_{\mathbf{a} \in \mathcal{V}_{K}}\left\|\mathrm{R}_{\frac{\pi}{2}} \nabla\left(\psi_{\mathbf{a}} u_{h}-s_{h}^{\mathbf{a}}\right)\right\|_{K}=\sum_{\mathbf{a} \in \mathcal{V}_{K}} \| \mathrm{R}_{\frac{\pi}{2}} \nabla\left(\psi_{\mathbf{a}} u_{h}\right)+\varsigma_{h}^{\mathbf{a}}\right) \|_{K} \\
& \leq \sum_{\mathbf{a} \in \mathcal{V}_{K}}\left\|\mathrm{R}_{\frac{\pi}{2}} \nabla\left(\psi_{\mathbf{a}} u_{h}\right)+\boldsymbol{\varsigma}_{h}^{\mathbf{a}}\right\|_{\omega_{\mathbf{a}}} .
\end{aligned}
$$

Noticing that (3.43) is equivalent to (3.39a) (and that $g^{\mathbf{a}}=0$ in this case, so that condition (3.39b) is trivially satisfied), Corollary 3.16 readily yields

$$
\left\|\mathrm{R}_{\frac{\pi}{2}} \nabla\left(\psi_{\mathbf{a}} u_{h}\right)+\boldsymbol{\varsigma}_{h}^{\mathbf{a}}\right\|_{\omega_{\mathbf{a}}} \leq C_{\mathrm{st}}\left\|\nabla r_{\mathbf{a}}\right\|_{\omega_{\mathbf{a}}}
$$

Lemma 3.13 concludes the proof of (3.44).

(2) The proof of (3.42) is similar, with the additional technicality of treating a possibly nonpolynomial source function $f$. Using Construction 3.4, $\left.\boldsymbol{\sigma}_{h}\right|_{K}=\left.\sum_{\mathbf{a} \in \mathcal{V}_{K}} \boldsymbol{\varsigma}_{h}^{\mathbf{a}}\right|_{K}$, the partition of unity (3.15), and the triangle inequality, we infer that

$$
\left\|\nabla u_{h}+\boldsymbol{\sigma}_{h}\right\|_{K}=\left\|\left.\sum_{\mathbf{a} \in \mathcal{V}_{K}}\left(\psi_{\mathbf{a}} \nabla u_{h}+\boldsymbol{\varsigma}_{h}^{\mathbf{a}}\right)\right|_{K}\right\|_{K} \leq \sum_{\mathbf{a} \in \mathcal{V}_{K}}\left\|\psi_{\mathbf{a}} \nabla u_{h}+\boldsymbol{\varsigma}_{h}^{\mathbf{a}}\right\|_{\omega_{\mathbf{a}}} .
$$

Note that replacing $\psi_{\mathbf{a}} f$ by $\Pi_{Q_{h}}\left(\psi_{\mathbf{a}} f\right)$ in $(3.12 \mathrm{~b})$ does not change the solution couple $\left(\boldsymbol{\varsigma}_{h}^{\mathbf{a}}, \bar{r}_{h}^{\mathbf{a}}\right)$ of $(3.10)$. Thus, setting $\tilde{g}^{\mathbf{a}}:=\Pi_{Q_{h}}\left(\psi_{\mathbf{a}} f\right)-\nabla \psi_{\mathbf{a}} \cdot \nabla u_{h}$, assumption (3.41b) implies (3.39b) with $g^{\mathbf{a}}$ replaced by $\tilde{g}^{\mathbf{a}}$, while assumption (3.41a) implies (3.39a). Consequently, Corollary 3.16 yields

$$
\left\|\psi_{\mathbf{a}} \nabla u_{h}+\boldsymbol{\varsigma}_{h}^{\mathbf{a}}\right\|_{\omega_{\mathbf{a}}} \leq C_{\mathrm{st}}\left\|\nabla \tilde{r}_{\mathbf{a}}\right\|_{\omega_{\mathbf{a}}}
$$


where $\tilde{r}_{\mathbf{a}}$ solves (3.24) with $g^{\mathbf{a}}$ replaced by $\tilde{g}^{\mathbf{a}}$. We now need to inspect the proof of Lemma 3.12 . We observe that

$$
\left(\nabla \tilde{r}_{\mathbf{a}}, \nabla v\right)_{\omega_{\mathbf{a}}}=-\left(\psi_{\mathbf{a}} \nabla u_{h}, \nabla v\right)_{\omega_{\mathbf{a}}}+\left(\psi_{\mathbf{a}} f-\nabla \psi_{\mathbf{a}} \cdot \nabla u_{h}, v\right)_{\omega_{\mathbf{a}}}+\left(\Pi_{Q_{h}}\left(\psi_{\mathbf{a}} f\right)-\psi_{\mathbf{a}} f, v\right)_{\omega_{\mathbf{a}}}
$$

in place of (3.28). The first two terms on the above right-hand side are treated as in the proof of Lemma 3.12, and we are left to bound

$$
\begin{aligned}
& \sup _{v \in H_{*}^{1}\left(\omega_{\mathbf{a}}\right) ;\|\nabla v\|_{\omega_{\mathbf{a}}}=1}\left(\Pi_{Q_{h}}\left(\psi_{\mathbf{a}} f\right)-\psi_{\mathbf{a}} f, v\right)_{\omega_{\mathbf{a}}} \\
= & \sup _{v \in H_{*}^{1}\left(\omega_{\mathbf{a}}\right) ;\|\nabla v\|_{\omega_{\mathbf{a}}}=1}\left\{\sum_{K^{\prime} \in \mathcal{T}_{\mathbf{a}}}\left(\Pi_{Q_{h}}\left(\psi_{\mathbf{a}} f\right)-\psi_{\mathbf{a}} f, v-\Pi_{K^{\prime}}^{0} v\right)_{K^{\prime}}\right\} \\
\leq & \left\{\sum_{K^{\prime} \in \mathcal{T}_{\mathbf{a}}}\left(\frac{h_{K^{\prime}}}{\pi}\left\|\psi_{\mathbf{a}} f-\Pi_{Q_{h}}\left(\psi_{\mathbf{a}} f\right)\right\|_{K^{\prime}}\right)^{2}\right\}^{1 / 2},
\end{aligned}
$$

as in (3.8). Combining the above results concludes the proof of (3.42).

Remark 3.18 (Data oscillation). As in Remark 3.6, if $f$ is elementwise smooth enough, the data oscillation term in (3.42) typically converges by two orders of magnitude faster than the energy error.

Remark 3.19 (Robustness for the potential reconstruction of Remark 3.11). Proceeding as in the above proof shows that under the assumptions

$$
\begin{aligned}
\left.\left(\psi_{\mathbf{a}} \mathrm{R}_{\frac{\pi}{2}} \nabla u_{h}\right)\right|_{K} \in \mathbf{V}_{h}(K) & \forall K \in \mathcal{T}_{\mathbf{a}}, \\
\left.\left(\left(\mathrm{R}_{\frac{\pi}{2}} \nabla \psi_{\mathbf{a}}\right) \cdot \nabla u_{h}\right)\right|_{K} \in Q_{h}(K) & \forall K \in \mathcal{T}_{\mathbf{a}},
\end{aligned}
$$

the potential reconstruction of Remark 3.11 satisfies the bound (3.44) with $C_{\mathrm{cont}, \mathrm{bPF}}$ replaced by $C_{\mathrm{cont}, \mathrm{P}}$ of (3.36).

Remark 3.20 (Examples of choice for the degree of $\mathbf{V}_{h}$ and $Q_{h}$ ). For $u_{h} \in \mathbb{P}_{p}\left(\mathcal{T}_{h}\right), p \geq 1$, as in many classical numerical methods on triangles, see examples in Section 4 below, an adequate choice for $\mathbf{V}_{h} \times Q_{h}$ is $\mathbf{R T}_{p} \times \mathbb{P}_{p}\left(\mathcal{T}_{h}\right)$. When $u_{h} \in \mathbb{Q}_{p}\left(\mathcal{T}_{h}\right), p \geq 1$, on rectangles, the choice $\mathbf{R T}_{p} \times \mathbb{Q}_{p}\left(\mathcal{T}_{h}\right)$ is adequate for (3.43) and (3.45), but $\mathbf{R T}_{p+1} \times \mathbb{Q}_{p+1}\left(\mathcal{T}_{h}\right)$ is necessary for (3.41).

Remark 3.21 (Reconstruction in Brezzi-Douglas-Marini spaces). The Brezzi-Douglas-Marini (BDM) spaces are given, on triangles for $p \geq 1$, by $\mathbf{B D M}_{p}:=\left\{\mathbf{v}_{h} \in \mathbf{H}(\operatorname{div}, \Omega) ;\left.\mathbf{v}_{h}\right|_{K} \in \mathbf{B D M}_{p}(K)\right\}$ and by $\mathbb{P}_{p-1}\left(\mathcal{T}_{h}\right)$, where $\operatorname{BDM}_{p}(K):=\left[\mathbb{P}_{p}(K)\right]^{2}$. For Corollary 3.16 to hold for such a choice of $\mathbf{V}_{h} \times Q_{h}$, one needs the polynomial-degree-robust right inverse of the divergence operator (for which numerical evidence is presented in [18]) and the BDM extension operator (which can be inferred from the RT case with divergence-free polynomials). In the case where $u_{h} \in \mathbb{P}_{p}\left(\mathcal{T}_{h}\right)$, the choice $\mathbf{B D M}_{p} \times \mathbb{P}_{p-1}\left(\mathcal{T}_{h}\right)$ is adequate to match conditions (3.41), (3.43), and (3.45); in this case, the data oscillation terms superconverge by only one order.

\subsection{Maximal overestimation}

Guaranteed (local) maximal overestimation factors have been derived previously in, e.g., Babuška et al. [15], Carstensen and Funken [24], Babuška and Strouboulis [14, Section 5.1], Prudhomme et al. [65], or Repin [68, Section 4.1.1], see also the references therein, but not necessarily simultaneously with a guaranteed upper bound. In our setting, we obtain:

Lemma 3.22 (Maximal overestimation). Let the assumptions of Theorem 3.17 be verified, with for simplicity $\psi_{\mathbf{a}} f \in Q_{h}$ (i.e., with (3.39b) satisfied). Then

$$
\begin{aligned}
\left\|\nabla u_{h}+\sigma_{h}\right\| & \leq 3 C_{\text {st }} C_{\text {cont }, \mathrm{PF}}\left\|\nabla\left(u-u_{h}\right)\right\|, \\
\left\|\nabla\left(u_{h}-s_{h}\right)\right\| & \leq 3 C_{\text {st }} C_{\text {cont }, \mathrm{bPF}}\left\|\nabla\left(u-u_{h}\right)\right\| .
\end{aligned}
$$


Proof. Employing $\left.\boldsymbol{\sigma}_{h}\right|_{K}=\left.\sum_{\mathbf{a} \in \mathcal{V}_{K}} \boldsymbol{\varsigma}_{h}^{\mathbf{a}}\right|_{K}$, the partition of unity (3.15), the Cauchy-Schwarz inequality, and proceeding as in the proof of Theorem 3.17, we infer that

$$
\begin{aligned}
& \left\|\nabla u_{h}+\sigma_{h}\right\|^{2} \\
= & \sum_{K \in \mathcal{T}_{h}}\left\|\nabla u_{h}+\boldsymbol{\sigma}_{h}\right\|_{K}^{2}=\sum_{K \in \mathcal{T}_{h}}\left\|\left.\sum_{\mathbf{a} \in \mathcal{V}_{K}}\left(\psi_{\mathbf{a}} \nabla u_{h}+\boldsymbol{\varsigma}_{h}^{\mathbf{a}}\right)\right|_{K}\right\|_{K}^{2} \\
\leq & 3 \sum_{K \in \mathcal{T}_{h}} \sum_{\mathbf{a} \in \mathcal{V}_{K}}\left\|\psi_{\mathbf{a}} \nabla u_{h}+\boldsymbol{s}_{h}^{\mathbf{a}}\right\|_{K}^{2}=3 \sum_{\mathbf{a} \in \mathcal{V}_{h}}\left\|\psi_{\mathbf{a}} \nabla u_{h}+\boldsymbol{\varsigma}_{h}^{\mathbf{a}}\right\|_{\omega_{\mathbf{a}}}^{2} \\
\leq & 3 C_{\mathrm{st}}^{2} C_{\mathrm{cont}, \mathrm{PF}}^{2} \sum_{\mathbf{a} \in \mathcal{V}_{h}}\left\|\nabla\left(u-u_{h}\right)\right\|_{\omega_{\mathbf{a}}}^{2}=9 C_{\mathrm{st}}^{2} C_{\mathrm{cont}, \mathrm{PF}}^{2}\left\|\nabla\left(u-u_{h}\right)\right\|^{2} .
\end{aligned}
$$

The bound for $\left\|\nabla\left(u_{h}-s_{h}\right)\right\|$ is similar.

We finally present a local result indicating additionally how to asses the value of the unknown constant $C_{\text {st }}$ of (3.40):

Lemma 3.23 (Guaranteed maximal local overestimation by auxiliary problems). Let the assumptions of Theorem 3.17 be verified, with additionally $\psi_{\mathbf{a}} f \in Q_{h}$. Fix $\mathbf{a} \in \mathcal{V}_{h}$ and consider an arbitrary conforming finite element approximation in $V_{h}^{\mathbf{a}}:=\mathbb{P}_{\bar{p}}\left(\mathcal{T}_{\mathbf{a}}\right) \cap H_{*}^{1}\left(\omega_{\mathbf{a}}\right)$ (or $\left.\mathbb{Q}_{\bar{p}}\left(\mathcal{T}_{\mathbf{a}}\right) \cap H_{*}^{1}\left(\omega_{\mathbf{a}}\right)\right), \bar{p} \geq 1$, of (3.24) or (3.32) in the form: find $r_{h}^{\mathbf{a}} \in V_{h}^{\mathbf{a}}$ such that

$$
\left(\nabla r_{h}^{\mathbf{a}}, \nabla v_{h}\right)_{\omega_{\mathbf{a}}}=-\left(\boldsymbol{\tau}_{h}^{\mathbf{a}}, \nabla v_{h}\right)_{\omega_{\mathbf{a}}}+\left(g^{\mathbf{a}}, v_{h}\right)_{\omega_{\mathbf{a}}} \quad \forall v_{h} \in V_{h}^{\mathbf{a}},
$$

with the usual choices (3.12) or (3.19) for the right-hand side. Then,

$$
\begin{gathered}
\left\|\psi_{\mathbf{a}} \nabla u_{h}+\varsigma_{h}^{\mathbf{a}}\right\|_{\omega_{\mathbf{a}}} \leq C_{\text {cont,PF }} \frac{\left\|\psi_{\mathbf{a}} \nabla u_{h}+\varsigma_{h}^{\mathbf{a}}\right\|_{\omega_{\mathbf{a}}}}{\left\|\nabla r_{h}^{\mathbf{a}}\right\|_{\omega_{\mathbf{a}}}}\left\|\nabla\left(u-u_{h}\right)\right\|_{\omega_{\mathbf{a}}}, \\
\left\|\nabla\left(\psi_{\mathbf{a}} u_{h}-s_{h}^{\mathbf{a}}\right)\right\|_{\omega_{\mathbf{a}}} \leq C_{\text {cont,bPF }} \frac{\left\|\nabla\left(\psi_{\mathbf{a}} u_{h}-s_{h}^{\mathbf{a}}\right)\right\|_{\omega_{\mathbf{a}}}}{\left\|\nabla r_{h}^{\mathbf{a}}\right\|_{\omega_{\mathbf{a}}}}\left\|\nabla\left(u-u_{h}\right)\right\|_{\omega_{\mathbf{a}}} .
\end{gathered}
$$

Proof. As $r_{h}^{\mathbf{a}}$ is the $(\nabla \cdot, \nabla \cdot)_{\omega_{\mathbf{a}}}$-orthogonal projection of $r_{\mathbf{a}}$ onto $V_{h}^{\mathbf{a}},\left\|\nabla r_{h}^{\mathbf{a}}\right\|_{\omega_{\mathbf{a}}} \leq\left\|\nabla r_{\mathbf{a}}\right\|_{\omega_{\mathbf{a}}}$. Thus the results follow respectively from Lemmas 3.12 and 3.13 .

Remark 3.24 (Size of overestimation, comparison with [24]). The above lemma together with Remark 3.15 suggest that the constant $C_{\mathrm{st}}$ approaches 1 as the polynomial degrees $p, \bar{p}$ are increased. Next, for convex patches $\omega_{\mathbf{a}}$ around interior vertices $\mathbf{a}, C_{\mathrm{P}, \omega_{\mathbf{a}}}=1 / \pi$, whereas $h_{\omega_{\mathbf{a}}}\left\|\nabla \psi_{\mathbf{a}}\right\|_{\infty, \omega_{\mathbf{a}}} \approx 2$ for "nice" meshes. Thus we may expect $C_{\text {cont,PF }} \approx 1+2 / \pi$ from the proof of Lemma 3.12 in such a case. Then Lemma 3.22 gives $3 C_{\mathrm{st}} C_{\mathrm{cont}, \mathrm{PF}} \approx 4.9$ for the maximal theoretical overestimation factor. In practice, however, the effectivity indices of the present estimates are quite close to the optimal value of one, see [18] and Section 5 below. For the conforming finite element method, Carstensen and Funken [24, Example 3.1] obtain a maximal theoretical overestimation factor 2.34 for "nice" meshes, which is roughly twice better than our result. This can be attributed to the localization of the estimators around mesh vertices with a specific use of the partition of unity in [24], see equation (3.7) in this reference and also the next remark, whereas we loose roughly a factor 3 in the estimate (3.46). Note, however, that the upper bound in [24] is, in contrast to the lower one, not guaranteed.

Remark 3.25 (Localization on the patches $\omega_{\mathbf{a}}$ ). In [24], see in particular Theorem 3.2 therein, the following local problems similar to (3.24) are considered: find $r_{\mathbf{a}} \in \bar{H}_{*}^{1}\left(\omega_{\mathbf{a}}\right)$ such that, with the choice (3.12) for the right-hand side,

$$
\left(\psi_{\mathbf{a}} \nabla r_{\mathbf{a}}, \nabla v\right)_{\omega_{\mathbf{a}}}=-\left(\boldsymbol{\tau}_{h}^{\mathbf{a}}, \nabla v\right)_{\omega_{\mathbf{a}}}+\left(g^{\mathbf{a}}, v\right)_{\omega_{\mathbf{a}}} \quad \forall v \in \bar{H}_{*}^{1}\left(\omega_{\mathbf{a}}\right),
$$

where $\bar{H}_{*}^{1}\left(\omega_{\mathbf{a}}\right)$ are $\psi_{\mathbf{a}}^{1 / 2}$-weighted versions of the spaces (3.25), and the (unfortunately not computable) a posteriori error estimator is simply $\left\|\psi_{\mathbf{a}}^{1 / 2} \nabla r_{\mathbf{a}}\right\|_{\omega_{\mathbf{a}}}$. Adjusting the equilibration of Construction 3.4, its computable upper bound may be constructed via local problems consisting in finding $\boldsymbol{\varsigma}_{h}^{\mathbf{a}} \in \mathbf{V}_{h}^{\mathbf{a}}$ and $\bar{r}_{h}^{\mathbf{a}} \in Q_{h}^{\mathbf{a}}$ such that

$$
\begin{array}{rlrl}
\left(\psi_{\mathbf{a}} \boldsymbol{\varsigma}_{h}^{\mathbf{a}}, \mathbf{v}_{h}\right)_{\omega_{\mathbf{a}}}- & \left(\bar{r}_{h}^{\mathbf{a}}, \nabla \cdot\left(\psi_{\mathbf{a}} \mathbf{v}_{h}\right)\right)_{\omega_{\mathbf{a}}}=-\left(\boldsymbol{\tau}_{h}^{\mathbf{a}}, \mathbf{v}_{h}\right)_{\omega_{\mathbf{a}}} & & \forall \mathbf{v}_{h} \in \mathbf{V}_{h}^{\mathbf{a}}, \\
\left(\nabla \cdot\left(\psi_{\mathbf{a}} \boldsymbol{S}_{h}^{\mathbf{a}}\right), q_{h}\right)_{\omega_{\mathbf{a}}}=\left(g^{\mathbf{a}}, q_{h}\right)_{\omega_{\mathbf{a}}} & \forall q_{h} \in Q_{h}^{\mathbf{a}}
\end{array}
$$




\section{Applications to discretization methods}

We show here how to apply our results to common discretizations via the verification of the assumptions of Section 3.

\subsection{Conforming finite elements}

Let, for $p \geq 1, V_{h}:=\mathbb{P}_{p}\left(\mathcal{T}_{h}\right) \cap H_{0}^{1}(\Omega)$ on triangles and $V_{h}:=\mathbb{Q}_{p}\left(\mathcal{T}_{h}\right) \cap H_{0}^{1}(\Omega)$ on rectangles. The conforming finite element method for (2.2), cf. [31], reads: find $u_{h} \in V_{h}$ such that

$$
\left(\nabla u_{h}, \nabla v_{h}\right)=\left(f, v_{h}\right) \quad \forall v_{h} \in V_{h} .
$$

The application of our framework is straightforward: (3.9) is nothing but the Galerkin orthogonality with respect to the hat basis function $\psi_{\mathbf{a}}$ which follows immediately from (4.1). The approximate solution $u_{h}$ is $H_{0}^{1}(\Omega)$-conforming, so that we set $s_{h}:=u_{h}$, the nonconformity estimators $\left\|\nabla\left(u_{h}-s_{h}\right)\right\|_{K}$ disappear, and there is nothing to verify in this respect. The resulting error estimators correspond to those of $[38,19,18]$.

\subsection{Nonconforming finite elements}

Let $V_{h}$ stand for functions from $\mathbb{P}_{p}\left(\mathcal{T}_{h}\right), p \geq 1$, on triangular meshes satisfying (3.31) for all polynomials up to degree $p-1$ on each edge instead of merely the constant function 1 . The nonconforming finite element method for (2.2), cf. Stoyan and Baran [71] or [9], reads: find $u_{h} \in V_{h}$ such that

$$
\left(\nabla u_{h}, \nabla v_{h}\right)=\left(f, v_{h}\right) \quad \forall v_{h} \in V_{h} .
$$

Nonconforming finite elements again fit perfectly into our framework: (3.9) follows immediately from (4.2) as $\psi_{\mathbf{a}} \in V_{h}$. The approximate solution $u_{h}$ is not included in $H_{0}^{1}(\Omega)$ but satisfies $(3.31)$ from the definition of the space $V_{h}$, so that both Construction 3.8 and that of Remark 3.11 are possible. For the lowest-order Crouzeix-Raviart case, the resulting error estimators are closely related to those of [29] for the potential reconstruction, whereas flux reconstructions by local mixed finite element problems (not polynomial-degree robust, on dual meshes) have already been proposed in [49].

Remark 4.1 (Implicit and explicit flux reconstructions). It has been recently shown in [46] that several seemingly different flux reconstructions for nonconforming finite elements coincide, including that of Construction 3.4 with the lowest-order RT space and the explicit constructions of [37, 2]. So, at least in this particular case, this smears the conceptual difference between the present implicit estimators (where solutions of local problems are necessary) and the, at first sight cheaper, explicit (directly computable) ones.

\subsection{Interior penalty discontinuous Galerkin (IPDG) finite elements}

Set $V_{h}:=\mathbb{P}_{p}\left(\mathcal{T}_{h}\right)$ on triangles and $V_{h}:=\mathbb{Q}_{p}\left(\mathcal{T}_{h}\right)$ on rectangles, $p \geq 1$, without any continuity requirement. The IPDG method, cf. [39] and the references therein, reads: find $u_{h} \in V_{h}$ such that

$$
\begin{aligned}
& \sum_{K \in \mathcal{T}_{h}}\left(\nabla u_{h}, \nabla v_{h}\right)_{K}-\sum_{e \in \mathcal{E}_{h}}\left\{\left\langle\left\{\left\{\nabla u_{h}\right\}\right\} \cdot \mathbf{n}_{e}, \llbracket v_{h} \rrbracket\right\rangle_{e}+\theta\left\langle\left\{\left\{\nabla v_{h}\right\}\right\} \cdot \mathbf{n}_{e}, \llbracket u_{h} \rrbracket\right\rangle_{e}\right\} \\
& +\sum_{e \in \mathcal{E}_{h}}\left\langle\alpha h_{e}^{-1} \llbracket u_{h} \rrbracket, \llbracket v_{h} \rrbracket\right\rangle_{e}=\left(f, v_{h}\right) \quad \forall v_{h} \in V_{h},
\end{aligned}
$$

where $\alpha$ is a positive stabilization parameter and $\theta \in\{-1,0,1\}$ corresponds respectively to the nonsymmetric, incomplete, and symmetric version. The present flux reconstruction has been recently introduced in [45, Section 6.4], while the present potential reconstruction is new. In $[16,3,52,41,32,43,42,10]$, see also the references therein, different elementwise flux reconstructions leading to (3.14) are designed. Although they are cheaper (typically no local linear system is to be solved), it is not clear whether they lead to polynomial-degree robust local efficiency. The same remark holds for the potential reconstruction, which is usually simply prescribed from nodal averages of the discrete solution. 


\subsubsection{Discrete gradient and flux reconstruction}

Introduce the discrete gradient $\mathfrak{G}\left(u_{h}\right):=\nabla u_{h}-\theta \sum_{e \in \mathcal{E}_{h}} \mathfrak{l}_{e}\left(\llbracket u_{h} \rrbracket\right)$ where the lifting operator $\mathfrak{l}_{e}: L^{2}(e) \rightarrow$ $\left[\mathbb{P}_{0}\left(\mathcal{T}_{h}\right)\right]^{2}$ is such that $\left.\left(\mathfrak{l}_{e}\left(\llbracket u_{h} \rrbracket\right), \mathbf{v}_{h}\right)=\left\langle\left\{\mathbf{v}_{h}\right\}\right\} \cdot \mathbf{n}_{e}, \llbracket u_{h} \rrbracket\right\rangle_{e}$ for all $\mathbf{v}_{h} \in\left[\mathbb{P}_{0}\left(\mathcal{T}_{h}\right)\right]^{2}$, see [39, Section 4.3]. Observe that $\mathfrak{G}(v)=\nabla v$ for any function $v$ with zero jumps or for any function in $H^{1}\left(\mathcal{T}_{h}\right)$ if $\theta=0$. Then, taking $v_{h}=\psi_{\mathbf{a}}$ in $(4.3)$ and since $\psi_{\mathbf{a}}$ has no jumps and $\nabla \psi_{\mathbf{a}} \in\left[\mathbb{P}_{0}\left(\mathcal{T}_{h}\right)\right]^{2}$, we infer that $\left(\mathfrak{G}\left(u_{h}\right), \nabla \psi_{\mathbf{a}}\right)_{\omega_{\mathbf{a}}}=\left(f, \psi_{\mathbf{a}}\right)_{\omega_{\mathbf{a}}}$ for all $\mathbf{a} \in \mathcal{V}_{h}^{\text {int }}$ instead of the hat-function orthogonality (3.9). Thus, Construction 3.4 for the flux is possible with right-hand sides $\tau_{h}^{\mathbf{a}}:=\psi_{\mathbf{a}} \mathfrak{G}\left(u_{h}\right)$ and $g^{\mathbf{a}}:=\psi_{\mathbf{a}} f-\nabla \psi_{\mathbf{a}} \cdot \mathfrak{G}\left(u_{h}\right)$. The guaranteed estimate of Theorem 3.3 using the discrete gradient takes the form

$$
\left\|\mathfrak{G}\left(u-u_{h}\right)\right\|^{2} \leq \sum_{K \in \mathcal{T}_{h}}\left(\left\|\mathfrak{G}\left(u_{h}\right)+\sigma_{h}\right\|_{K}+\frac{h_{K}}{\pi}\left\|f-\Pi_{Q_{h}} f\right\|_{K}\right)^{2}+\sum_{K \in \mathcal{T}_{h}}\left\|\mathfrak{G}\left(u_{h}-s_{h}\right)\right\|_{K}^{2},
$$

and the local efficiency result (3.42) for the flux reconstruction (with $\psi_{\mathbf{a}} f \in Q_{h}$ for simplicity) takes the form

$$
\left\|\mathfrak{G}\left(u_{h}\right)+\sigma_{h}\right\|_{K} \leq C_{\mathrm{st}} C_{\text {cont }, \mathrm{PF}} \sum_{\mathbf{a} \in \mathcal{V}_{K}}\left\|\mathfrak{G}\left(u-u_{h}\right)\right\|_{\omega_{\mathbf{a}}},
$$

with the polynomial-degree-independent constants $C_{\text {st }}$ of (3.40) and $C_{\text {cont,PF }}$ of (3.26).

\subsubsection{Potential reconstruction for the nonsymmetric and incomplete versions}

We use Construction 3.8 for the potential reconstruction (observe that condition (3.22) does not hold). As the mean-zero condition (3.31) on the jumps is not satisfied either, we cannot use directly Lemma 3.13. The inspection of its proof, however, shows that we merely need to replace the estimate (3.35) by

$$
\begin{aligned}
\left\|\nabla\left(\psi_{\mathbf{a}} \tilde{u}-\psi_{\mathbf{a}} u_{h}\right)\right\|_{\omega_{\mathbf{a}} \leq} \leq & \left\|\nabla \psi_{\mathbf{a}}\right\|_{\infty, \omega_{\mathbf{a}}}\left\|\tilde{u}-u_{h}\right\|_{\omega_{\mathbf{a}}}+\left\|\psi_{\mathbf{a}}\right\|_{\infty, \omega_{\mathbf{a}}}\left\|\nabla\left(\tilde{u}-u_{h}\right)\right\|_{\omega_{\mathbf{a}}} \\
\leq & \left(1+C_{\mathrm{bPF}, \omega_{\mathbf{a}}} h_{\omega_{\mathbf{a}}}\left\|\nabla \psi_{\mathbf{a}}\right\|_{\infty, \omega_{\mathbf{a}}}\right)\left\|\nabla\left(\tilde{u}-u_{h}\right)\right\|_{\omega_{\mathbf{a}}} \\
& +C_{\mathrm{bPF}, \omega_{\mathbf{a}}} h_{\omega_{\mathbf{a}}}\left\|\nabla \psi_{\mathbf{a}}\right\|_{\infty, \omega_{\mathbf{a}}}\left\{\sum_{e \in \mathcal{E}_{h}^{\text {int }}, \mathbf{a} \in e} h_{e}^{-1}\left\|\Pi_{e}^{0} \llbracket u_{h} \rrbracket\right\|_{e}^{2}\right\}^{1 / 2},
\end{aligned}
$$

with $\Pi_{e}^{0}$ the $L^{2}(e)$-orthogonal projection onto constants, using the discrete Poincaré-Friedrichs inequalities of [20, Remark 1.1] (since $\left(\tilde{u}-u_{h}, 1\right)_{\omega_{\mathbf{a}}}=0$ and since $\tilde{u}$ has no jumps). Thus,

$$
\left\|\nabla r_{\mathbf{a}}\right\|_{\omega_{\mathbf{a}}} \leq C_{\text {cont }, \mathrm{bPF}}\left(\left\|\nabla\left(u-u_{h}\right)\right\|_{\omega_{\mathbf{a}}}+\left\{\sum_{e \in \mathcal{E}_{h}^{\text {int }}, \mathbf{a} \in e} h_{e}^{-1}\left\|\Pi_{e}^{0} \llbracket u-u_{h} \rrbracket\right\|_{e}^{2}\right\}^{1 / 2}\right)
$$

in place of (3.34). The local efficiency result (3.44) then yields

$$
\left\|\nabla\left(u_{h}-s_{h}\right)\right\|_{K} \leq C_{\mathrm{st}} C_{\text {cont, bPF }} \sum_{\mathbf{a} \in \mathcal{V}_{K}}\left(\left\|\nabla\left(u-u_{h}\right)\right\|_{\omega_{\mathbf{a}}}+\left\{\sum_{e \in \mathcal{E}_{h}^{\text {int }}, \mathbf{a} \in e} h_{e}^{-1}\left\|\Pi_{e}^{0} \llbracket u-u_{h} \rrbracket\right\|_{e}^{2}\right\}^{1 / 2}\right) .
$$

It is still polynomial-degree robust, but features the additional jump term. The classical option to obtain both upper and lower bounds for the same error measure is to resort to the jumps-augmented energy norm, thereby replacing (4.4) by

$$
\begin{aligned}
\left\|\mathfrak{G}\left(u-u_{h}\right)\right\|^{2}+\sum_{e \in \mathcal{E}_{h}} h_{e}^{-1}\left\|\Pi_{e}^{0} \llbracket u-u_{h} \rrbracket\right\|_{e}^{2} \leq & \sum_{K \in \mathcal{T}_{h}}\left(\left\|\mathfrak{G} u_{h}+\sigma_{h}\right\|_{K}+\frac{h_{K}}{\pi}\left\|f-\Pi_{Q_{h}} f\right\|_{K}\right)^{2} \\
& +\sum_{K \in \mathcal{T}_{h}}\left\|\mathfrak{G}\left(u_{h}-s_{h}\right)\right\|_{K}^{2}+\sum_{e \in \mathcal{E}_{h}} h_{e}^{-1}\left\|\Pi_{e}^{0} \llbracket u_{h} \rrbracket\right\|_{e}^{2},
\end{aligned}
$$

using that $\llbracket u-u_{h} \rrbracket=-\llbracket u_{h} \rrbracket$. Then, for the incomplete version, observing that $\nabla\left(u_{h}-s_{h}\right)=\mathfrak{G}\left(u_{h}-s_{h}\right)$ and $\nabla\left(u-u_{h}\right)=\mathfrak{G}\left(u-u_{h}\right)$ in (4.6), (4.5) combined with (4.6) yields polynomial-degree-robust local efficiency for the same error measure as in (4.7). 
For the nonsymmetric version, we need a bound similar to (4.6), but using the discrete gradient. Since the lifting $\mathfrak{l}$ only includes the neighboring elements and using the triangle inequality, we infer that

$$
\left\|\mathfrak{G}\left(u_{h}-s_{h}\right)\right\|_{K} \leq\left\|\nabla\left(u_{h}-s_{h}\right)\right\|_{K}+\sum_{e \in \mathcal{E}_{K}}\left\|\mathfrak{l}_{e}\left(\llbracket u_{h} \rrbracket\right)\right\|_{K}
$$

The term $\left\|\nabla\left(u_{h}-s_{h}\right)\right\|_{K}$ is bounded using (4.6), where on the right-hand side, we further bound $\| \nabla(u-$ $\left.u_{h}\right) \|_{\omega_{\mathbf{a}}}$ by $\left\|\mathfrak{G}\left(u-u_{h}\right)\right\|_{\omega_{\mathbf{a}}}+\sum_{K \in \mathcal{T}_{\mathbf{a}}} \sum_{e \in \mathcal{E}_{K}}\left\|\mathfrak{l}_{e}\left(\llbracket u_{h} \rrbracket\right)\right\|_{K}$. Additionally, relying on the fact that the lifting $\mathfrak{l}$ maps onto piecewise constant functions,

$$
\begin{aligned}
\left\|\mathfrak{l}_{e}\left(\llbracket u_{h} \rrbracket\right)\right\|_{K} & \leq \sup _{\mathbf{v}_{h} \in\left[\mathbb{P}_{0}\left(\mathcal{T}_{e}\right)\right]^{2} ;\left\|\mathbf{v}_{h}\right\|_{\mathcal{T}_{e}=1}}\left(\mathfrak{l}_{e}\left(\llbracket u_{h} \rrbracket\right), \mathbf{v}_{h}\right)_{\mathcal{T}_{e}} \\
& =\sup _{\mathbf{v}_{h} \in\left[\mathbb{P}_{0}\left(\mathcal{T}_{e}\right)\right]^{2} ;\left\|\mathbf{v}_{h}\right\|_{\mathcal{T}_{e}=1}}\left\langle\left\{\left\{\mathbf{v}_{h}\right\}\right\} \cdot \mathbf{n}_{e}, \Pi_{e}^{0} \llbracket u-u_{h} \rrbracket\right\rangle_{e} \\
& \leq C_{\kappa \mathcal{T}} h_{e}^{-1 / 2}\left\|\Pi_{e}^{0} \llbracket u-u_{h} \rrbracket\right\|_{e},
\end{aligned}
$$

where $\mathcal{T}_{e}$ stands for the (one or two) elements sharing the edge $e$ and $C_{\kappa_{\mathcal{T}}}$ uniformly bounds $\frac{h_{e}}{|K|^{1 / 2}}$ and only depends on the mesh-regularity parameter $\kappa_{\mathcal{T}}$. Finally,

$$
\left\|\mathfrak{G}\left(u_{h}-s_{h}\right)\right\|_{K} \leq C_{\mathrm{st}} C_{\mathrm{cont}, \mathrm{bPF}} \sum_{\mathbf{a} \in \mathcal{V}_{K}}\left\|\mathfrak{G}\left(u-u_{h}\right)\right\|_{\omega_{\mathbf{a}}}+C\left\{\sum_{e \in \mathcal{E}_{K}^{+}} h_{e}^{-1}\left\|\Pi_{e}^{0} \llbracket u-u_{h} \rrbracket\right\|_{e}^{2}\right\}^{1 / 2},
$$

where $C$ only depends on the mesh-regularity parameter $\kappa_{\mathcal{T}}$ and $\mathcal{E}_{K}^{+}:=\left\{e \in \mathcal{E}_{h} \mid \exists \mathbf{a} \in \mathcal{V}_{K}, \exists K^{\prime} \in \mathcal{T}_{\mathbf{a}}, e \in \mathcal{E}_{K^{\prime}}\right\}$, so that (4.5) combined with (4.10) yields polynomial-degree-robust local efficiency for the same error measure as in (4.7).

\subsubsection{Potential reconstruction for the symmetric version}

A remarkable fact is that the discrete gradient $\mathfrak{G}$ satisfies the following modification of condition (3.22) related to the alternative potential reconstruction from Remark 3.11:

$$
\left(\mathfrak{G}\left(u_{h}\right), \mathrm{R}_{\frac{\pi}{2}} \nabla \psi_{\mathbf{a}}\right)_{\omega_{\mathbf{a}}}=0 \quad \forall \mathbf{a} \in \mathcal{V}_{h}
$$

Indeed, using the definition of the discrete gradient and the Green theorem, we have

$$
\begin{aligned}
\left(\mathfrak{G}\left(u_{h}\right), \mathrm{R}_{\frac{\pi}{2}} \nabla \psi_{\mathbf{a}}\right)_{\omega_{\mathbf{a}}} & =\left(\nabla u_{h}, \mathrm{R}_{\frac{\pi}{2}} \nabla \psi_{\mathbf{a}}\right)_{\omega_{\mathbf{a}}}-\theta \sum_{e \in \mathcal{E}_{h}}\left\langle\left\{\left\{\mathrm{R}_{\frac{\pi}{2}} \nabla \psi_{\mathbf{a}}\right\} \cdot \mathbf{n}_{e}, \llbracket u_{h} \rrbracket\right\rangle_{e}\right. \\
& =\sum_{K \in \mathcal{T}_{\mathbf{a}}}\left\langle u_{h},\left(\mathrm{R}_{\frac{\pi}{2}} \nabla \psi_{\mathbf{a}}\right) \cdot \mathbf{n}_{K}\right\rangle_{\partial K}-\theta \sum_{e \in \mathcal{E}_{h}}\left\langle\left\{\left\{\mathrm{R}_{\frac{\pi}{2}} \nabla \psi_{\mathbf{a}}\right\} \cdot \mathbf{n}_{e}, \llbracket u_{h} \rrbracket\right\rangle_{e} .\right.
\end{aligned}
$$

Now for $\theta=1$, the two above terms cancel. Thus we can use here the procedure of Remark 3.11 , where we systematically replace $\nabla v$ by $\mathfrak{G}(v)$. The local efficiency result for the flux reconstruction is $(4.5)$ and the one for the potential reconstruction takes the form discussed in Remark 3.19,

$$
\left\|\mathfrak{G}\left(u_{h}-s_{h}\right)\right\|_{K} \leq C_{\text {st }} C_{\text {cont }, \mathrm{P}} \sum_{\mathbf{a} \in \mathcal{V}_{K}}\left\|\mathfrak{G}\left(u-u_{h}\right)\right\|_{\omega_{\mathbf{a}}}
$$

with the polynomial-degree-independent constants $C_{\text {st }}$ of (3.40) and $C_{\text {cont,P }}$ of (3.36). Note that in this symmetric case, the lifting operator $\mathfrak{l}$ can alternatively be designed as $\mathfrak{l}_{e}: L^{2}(e) \rightarrow\left[\mathbb{P}_{p-1}\left(\mathcal{T}_{h}\right)\right]^{2}$ on triangles or $\left[\mathbb{Q}_{p-1}\left(\mathcal{T}_{h}\right)\right]^{2}$ on rectangles with $\left(\mathfrak{l}_{e}\left(\llbracket u_{h} \rrbracket\right), \mathbf{v}_{h}\right)=\left\langle\left\{\left\{\mathbf{v}_{h}\right\}\right\} \cdot \mathbf{n}_{e}, \llbracket u_{h} \rrbracket\right\rangle_{e}$ for all $\mathbf{v}_{h} \in\left[\mathbb{P}_{p-1}\left(\mathcal{T}_{h}\right)\right]^{2}$ or $\left[\mathbb{Q}_{p-1}\left(\mathcal{T}_{h}\right)\right]^{2}$.

\subsection{Mixed finite elements}

The application of our framework to mixed finite elements is again rather straightforward. Let $\mathbf{V}_{h} \times Q_{h}$ be any of the usual mixed finite element spaces, see Section 2.4 and Remark 3.21; we consider here the polynomial degree $p^{\prime} \geq 0$. We look for the couple $\boldsymbol{\sigma}_{h} \in \mathbf{V}_{h}$ and $\bar{u}_{h} \in Q_{h}$ such that, cf. [21, 69, 78],

$$
\begin{aligned}
\left(\boldsymbol{\sigma}_{h}, \mathbf{v}_{h}\right)-\left(\bar{u}_{h}, \nabla \cdot \mathbf{v}_{h}\right) & =0 & & \forall \mathbf{v}_{h} \in \mathbf{V}_{h}, \\
\left(\nabla \cdot \boldsymbol{\sigma}_{h}, q_{h}\right) & =\left(f, q_{h}\right) & & \forall q_{h} \in Q_{h} .
\end{aligned}
$$


We have written the formulation explicitly with $\boldsymbol{\sigma}_{h}$ since this computed flux can serve directly as the equilibrated flux reconstruction of Definition 3.1. Flux equilibration following Construction 3.4 is useless here (and unfeasible as (3.9) does not hold true in general); remark also that we directly have (3.14) by $(4.12 \mathrm{~b})$.

The original potential approximation $\bar{u}_{h}$ has low regularity (it is only piecewise constant in lowest-order methods); local postprocessing is usually employed to improve it. In particular, following Arnold and Brezzi [12], Arbogast and Chen [11], and [76], there exists for each couple $\mathbf{V}_{h} \times Q_{h}$ a piecewise polynomial space $M_{h}$ such that $u_{h} \in M_{h}$ can be prescribed by

$$
\begin{aligned}
\Pi_{Q_{h}(K)}\left(\left.u_{h}\right|_{K}\right) & =\left.\bar{u}_{h}\right|_{K} & & \forall K \in \mathcal{T}_{h}, \\
\Pi_{\mathbf{V}_{h}(K)}\left(\left.\left(-\nabla u_{h}\right)\right|_{K}\right) & =\left.\sigma_{h}\right|_{K} & & \forall K \in \mathcal{T}_{h},
\end{aligned}
$$

where $\Pi_{Q_{h}(K)}$ is the $L^{2}(K)$-orthogonal projection on $Q_{h}(K)$ and $\Pi_{\mathbf{V}_{h}(K)}$ is the $\left[L^{2}(K)\right]^{2}$-orthogonal projection on $\mathbf{V}_{h}(K)$. Plugging (4.13) into (4.12a), it follows

$$
-\left(\nabla u_{h}, \mathbf{v}_{h}\right)-\left(u_{h}, \nabla \cdot \mathbf{v}_{h}\right)=0 \quad \forall \mathbf{v}_{h} \in \mathbf{V}_{h}
$$

An immediate consequence of the Green theorem and the structure of $\mathbf{V}_{h}$ is that

$$
\left\langle\llbracket u_{h} \rrbracket, v_{h}\right\rangle_{e}=0 \quad \forall e \in \mathcal{E}_{h}, \forall v_{h} \in \mathbf{V}_{h} \cdot \mathbf{n}_{e}(e),
$$

i.e., the jumps of $u_{h}$ are orthogonal to all polynomials from $\mathbf{V}_{h} \cdot \mathbf{n}$. We let $p$ denote the polynomial degree of functions in $M_{h}$, so that $u_{h}$, as throughout this paper, is a $p$-degree piecewise polynomial. With respect to the present a posteriori analysis, the crucial feature is that (4.14) implies (3.31).

For $u_{h}$ from (4.13), the upper bound of Theorem 3.3 holds true, with $\boldsymbol{\sigma}_{h}$ obtained directly from (4.12) and $s_{h}$ from Construction 3.8 or from Remark 3.11. The local lower bound (3.44) holds true but (3.42) cannot be verified, as $\boldsymbol{\sigma}_{h}$ was not derived from $u_{h}$ by Construction 3.4. This, fortunately, is not obstructive, as $\left\|\nabla u_{h}+\sigma_{h}\right\|$ by (4.13b) takes small values and can be seen as a numerical quadrature. Alternatively, proceeding as in [78], we may estimate simultaneously the error in both the flux and potential approximations $\boldsymbol{\sigma}_{h}$ and $u_{h}$. This yields

$$
\begin{aligned}
\left\|\nabla\left(u-u_{h}\right)\right\|^{2}+\left\|\nabla u+\boldsymbol{\sigma}_{h}\right\|^{2} \leq & \sum_{K \in \mathcal{T}_{h}}\left(\left\|\nabla u_{h}+\boldsymbol{\sigma}_{h}\right\|_{K}+\frac{h_{K}}{\pi}\left\|f-\Pi_{Q_{h}} f\right\|_{K}\right)^{2} \\
& +\sum_{K \in \mathcal{T}_{h}}\left\|\nabla\left(u_{h}-s_{h}\right)\right\|_{K}^{2} \\
& +\sum_{K \in \mathcal{T}_{h}}\left\|\nabla s_{h}+\boldsymbol{\sigma}_{h}\right\|_{K}^{2}+\sum_{K \in \mathcal{T}_{h}}\left(\frac{h_{K}}{\pi}\left\|f-\Pi_{Q_{h}} f\right\|_{K}\right)^{2} .
\end{aligned}
$$

The efficiency result is then derived by using (3.44) for $\left\|\nabla\left(u_{h}-s_{h}\right)\right\|_{K},\left\|\nabla s_{h}+\sigma_{h}\right\|_{K} \leq\left\|\nabla\left(u_{h}-s_{h}\right)\right\|_{K}+$ $\left\|\nabla u_{h}+\boldsymbol{\sigma}_{h}\right\|_{K}$, and $\left\|\nabla u_{h}+\boldsymbol{\sigma}_{h}\right\|_{K} \leq\left\|\nabla\left(u-u_{h}\right)\right\|_{K}+\left\|\nabla u+\boldsymbol{\sigma}_{h}\right\|_{K}$. The resulting error estimators are, to the authors' knowledge, new, and are the first ones to deliver a provable polynomial-degree robust local efficiency.

\section{Numerical illustration}

A numerical illustration is provided in this section. We consider problem $(2.1)$ with $\Omega=(0,1) \times(0,1)$ and the right-hand side such that the exact solution is $u(x, y)=\sin (2 \pi x) \sin (2 \pi y)$. The discretization is performed via the incomplete interior penalty discontinuous Galerkin method (4.3) with $\theta=0$ and $\alpha=20$ (following Dolejší [40]), where we vary the polynomial degree $p$ between 1 and 5 . We consider an unstructured triangular mesh of $\Omega$ with the initial mesh size $h_{0}:=0.168$ that we refine uniformly (every triangle is divided into 4 congruent triangles) three times. The equilibrated flux $\boldsymbol{\sigma}_{h}$ is obtained via Construction 3.4 and the potential $s_{h}$ via Construction 3.8. In both cases, we consider Raviart-Thomas equilibrations of degree $p$, $\mathbf{V}_{h} \times Q_{h}:=\mathbf{R T}_{p} \times \mathbb{P}_{p}\left(\mathcal{T}_{h}\right)$.

Table 1 reports the energy seminorm $\left\|\nabla\left(u-u_{h}\right)\right\|$, the jump seminorm $\left\|u-u_{h}\right\|_{\mathrm{J}}^{2}:=\sum_{e \in \mathcal{E}_{h}} h_{e}^{-1} \| \Pi_{e}^{0} \llbracket u-$ $u_{h} \rrbracket \|_{e}^{2}$, the full DG norm $\left\|u-u_{h}\right\|_{\mathrm{DG}}^{2}:=\left\|\nabla\left(u-u_{h}\right)\right\|^{2}+\left\|u-u_{h}\right\|_{\mathrm{J}}^{2}$, the estimator $\eta$ corresponding to (3.3), the 


\begin{tabular}{|c|c|c|c|c|c|c|c|c|c|}
\hline$P \mid$ & $n \pi$ & $u-w_{h \| J}$ & $\mid u-u_{h} \| \mathrm{DG}$ & $\overline{u_{h}+\sigma_{h}}$ & $\| \nabla\left(u_{h}-s_{h}\right)$ & & 7 & & \\
\hline & $1.21 \mathrm{E}+00$ & $1.44 \mathrm{E}-01$ & $1.22 \mathrm{E}+00$ & $1.24 \mathrm{E}+00$ & & & (T) & & \\
\hline & $\begin{array}{r}6.18 \mathrm{H} \\
(0.9\end{array}$ & $\begin{array}{r}6.95 \\
(1 .\end{array}$ & & $(0$. & & & 01 & $(1$ & 1 \\
\hline$\frac{\frac{10}{4}}{4}$ & $\begin{array}{c}3.12 \mathrm{E}-01 \\
(0.99)\end{array}$ & $\begin{array}{r}3.41 \mathrm{~F} \\
(1.0\end{array}$ & $\begin{array}{c}3.13 \mathrm{E}-01 \\
(0.99)\end{array}$ & $\begin{array}{r}3.22 \\
(0 .\end{array}$ & $\begin{array}{r}2.43 \\
(1\end{array}$ & & $\begin{array}{c}3.24 \mathrm{E}-01 \\
(1.00)\end{array}$ & $\begin{array}{r}3.25 \\
(1\end{array}$ & 1 \\
\hline$\frac{20}{8}$ & $\begin{array}{r}1.56 \mathrm{E} \\
(1.0\end{array}$ & $\begin{array}{r}1.69 \mathrm{~F} \\
(1.0\end{array}$ & $\begin{array}{r}1.57 \mathrm{I} \\
(1 . \mathrm{C}\end{array}$ & & & & & & 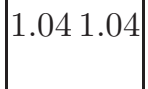 \\
\hline & 1.50 & & & & & & & & \\
\hline & $\begin{array}{r}3.85 \\
(1 .\end{array}$ & (1 & $(1$ & & & & & & \\
\hline$\underline{0}$ & $\begin{array}{r}9.701 \\
(1 .\end{array}$ & $\begin{array}{r}1.91 \\
(1 .\end{array}$ & & & & & & & \\
\hline$\frac{h_{0}}{8}$ & $\begin{array}{r}2.43 \\
(1 .\end{array}$ & $\begin{array}{r}4.82 \\
(1\end{array}$ & $\begin{array}{r}2.48 \\
(1\end{array}$ & & & & & & 1 \\
\hline $\mid h_{0} 3$ & 1.32 & 3 & 12 & & & & & & $\mathrm{~T}$ \\
\hline$\frac{h_{0}}{2}$ & & (3 & $(2$ & & & & & & \\
\hline$\frac{h_{0}}{4}$ & $\begin{array}{r}2.11 \\
(2 .\end{array}$ & $\begin{array}{r}3.12 \\
(3\end{array}$ & $\begin{array}{r}2.1 \\
(2\end{array}$ & & & & & & \\
\hline$\frac{h_{0}}{8}$ & $\begin{array}{r}2.64 \\
(3 \\
\end{array}$ & $\begin{array}{r}3.85 \\
(3 \\
\end{array}$ & $\begin{array}{r}2.67 \\
(3 \\
\end{array}$ & & & & & & 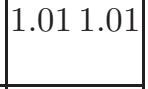 \\
\hline & 9.36 & 184 & & & & & 9.5 & 04 & \\
\hline$\frac{h_{0}}{2}$ & $\begin{array}{r}5.93 \\
(3 .\end{array}$ & $\begin{array}{r}1.22 \\
(3 .\end{array}$ & $\begin{array}{r}6.0 \\
(3\end{array}$ & $\begin{array}{r}5.7 \\
(3\end{array}$ & & & & & \\
\hline$\frac{h_{0}}{4}$ & $\begin{array}{c}3.72 \mathrm{E}-06 \\
(3.99)\end{array}$ & $\begin{array}{r}7.76 \mathrm{1} \\
(3 .\end{array}$ & $\begin{array}{r}3.80 \\
(3 .\end{array}$ & & 1.1 & $\begin{array}{r}5.3 \\
(5\end{array}$ & $\begin{array}{r}3.80 \\
(3 .\end{array}$ & $\begin{array}{r}3.87 \\
(3\end{array}$ & 1.021 .02 \\
\hline$\frac{h_{0}}{8}$ & $\begin{array}{c}2.33 \mathrm{E}-07 \\
(4.00)\end{array}$ & $\begin{array}{c}4.90 \mathrm{E}-08 \\
(3.99)\end{array}$ & $\begin{array}{c}2.38 \mathrm{E}-07 \\
(4.00)\end{array}$ & $\begin{array}{r}2.27 \\
(4 .\end{array}$ & $\begin{array}{r}7.0 \\
(3\end{array}$ & $\begin{array}{c}8.30 \mathrm{E}-11 \\
(6.00)\end{array}$ & $\begin{array}{c}2.38 \mathrm{E}-07 \\
(4.00)\end{array}$ & $\begin{array}{c}2.43 \mathrm{E}-07 \\
(3.99)\end{array}$ & 1.021 .02 \\
\hline & & & & & & & & & \\
\hline$h_{0}$ & 1.7 & $\begin{array}{r}2.97 \\
(5\end{array}$ & 1. & & & & -06 & & \\
\hline 望 & & $\begin{array}{r}9.10 \\
(5 .\end{array}$ & & & & & & & 1.011. \\
\hline$\frac{\frac{n}{8}}{8}$ & $\begin{array}{c}1.66 \mathrm{E}-09 \\
(5.00)\end{array}$ & $\begin{array}{c}2.81 \mathrm{E}-10 \\
(5.02)\end{array}$ & $\begin{array}{c}1.69 \mathrm{E}-09 \\
(5.00)\end{array}$ & $\begin{array}{r}1.62 \mathrm{E}-0 \\
(5.00) \\
\end{array}$ & $\begin{array}{r}4.4 \\
(4 \\
\end{array}$ & $\begin{array}{c}5.99 \mathrm{E}-13 \\
(7.00) \\
\end{array}$ & $\begin{array}{c}1.68 \mathrm{E}-09 \\
(5.00)\end{array}$ & $\begin{array}{c}1.70 \mathrm{E}-09 \\
(5.00) \\
\end{array}$ & 1.01 \\
\hline
\end{tabular}

Table 1: Numerical results for a smooth solution $\sin (2 \pi x) \sin (2 \pi y)$ on a unit square and the incomplete interior penalty discontinuous Galerkin method

full DG estimator $\eta_{\mathrm{DG}}^{2}:=\eta^{2}+\left\|u_{h}\right\|_{\mathrm{J}}^{2}$ of (4.7), as well as the individual estimators $\left\|\nabla u_{h}+\boldsymbol{\sigma}_{h}\right\|,\left\|\nabla\left(u_{h}-s_{h}\right)\right\|$, and the data oscillation $\eta_{\text {osc }}^{2}:=\sum_{K \in \mathcal{T}_{h}}\left(\frac{h_{K}}{\pi}\left\|f-\nabla \cdot \boldsymbol{\sigma}_{h}\right\|_{K}\right)^{2}$. The table also reports the effectivity indices (overestimation factors) $I^{\mathrm{eff}}:=\frac{\eta}{\left\|\nabla\left(u-u_{h}\right)\right\|}$ and $I_{\mathrm{DG}}^{\text {eff }}:=\frac{\eta_{\mathrm{DG}}}{\left\|u-u_{h}\right\|_{\mathrm{DG}}}$ and the corresponding experimental orders of convergence (in parentheses). As predicted by the theory, the estimators $\eta$ and $\eta_{\mathrm{DG}}$ deliver guaranteed upper bounds on the respective errors, with the effectivity indices independent of the polynomial degree $p$. Moreover, we experimentally observe asymptotic exactness for this smooth solution case.

\section{Concluding remarks}

For any numerical method approximating (2.1), the stiffness matrix needs to be assembled. In the present a posteriori error estimates, we need to similarly assemble the block-diagonal matrix with $(3.10) /(3.17)$ for 
each mesh vertex. Then the computation of the degrees of freedom of the flux and potential reconstructions corresponds to solving a block-diagonal system (or to a matrix-vector multiplication if the inverse of the block-diagonal matrix is pre-processed). Similarly, the actual evaluation of the estimators of Theorem 3.3 can be implemented as a matrix-vector multiplication formula stemming from the appropriate quadrature rule and the above degrees of freedom. Thus, the slightly increased cost of this approach seems to be largely compensated by its advantages: it offers a unified setting for a large spectrum of numerical methods, a guaranteed upper bound, a lower bound robust with respect to polynomial degree, very moderate overestimation factors, and no parameter to tune. Moreover, different error components can be distinguished, see [45] and the references therein, leading to fully adaptive strategies with adaptive stopping criteria for linear and nonlinear solvers, adaptive time step choice, and adaptive mesh refinement. The present theory extends to $d$ space dimensions, $d \geq 3$, except for the potential reconstructions, which are the subject of ongoing work. Further numerical experiments as a part of $h p$-refinement strategies are in preparation.

\section{Acknowledgment}

The authors are thankful to V. Dolejší (Charles University in Prague) for kindly providing the numerical results.

\section{References}

[1] A. Agouzal, A posteriori error estimator for nonconforming finite element methods, Appl. Math. Lett., 7 (1994), pp. 61-66.

[2] M. Ainsworth, Robust a posteriori error estimation for nonconforming finite element approximation, SIAM J. Numer. Anal., 42 (2005), pp. 2320-2341.

[3] — A posteriori error estimation for discontinuous Galerkin finite element approximation, SIAM J. Numer. Anal., 45 (2007), pp. 1777-1798.

[4] - A posteriori error estimation for lowest order Raviart-Thomas mixed finite elements, SIAM J. Sci. Comput., 30 (2007), pp. 189-204.

[5] — A framework for obtaining guaranteed error bounds for finite element approximations, J. Comput. Appl. Math., 234 (2010), pp. 2618-2632.

[6] M. Ainsworth And X. MA, Non-uniform order mixed FEM approximation: Implementation, postprocessing, computable error bound and adaptivity, J. Comput. Phys., 231 (2012), pp. 436-453.

[7] M. Ainsworth And J. T. Oden, A unified approach to a posteriori error estimation using element residual methods, Numer. Math., 65 (1993), pp. 23-50.

[8] — - A posteriori error estimation in finite element analysis, Pure and Applied Mathematics (New York), Wiley-Interscience [John Wiley \& Sons], New York, 2000.

[9] M. Ainsworth And R. Rankin, Fully computable bounds for the error in nonconforming finite element approximations of arbitrary order on triangular elements, SIAM J. Numer. Anal., 46 (2008), pp. 3207-3232.

[10] - Constant free error bounds for nonuniform order discontinuous Galerkin finite-element approximation on locally refined meshes with hanging nodes, IMA J. Numer. Anal., 31 (2011), pp. 254-280.

[11] T. Arbogast And Z. Chen, On the implementation of mixed methods as nonconforming methods for second-order elliptic problems, Math. Comp., 64 (1995), pp. 943-972.

[12] D. N. ARnold And F. Brezzi, Mixed and nonconforming finite element methods: implementation, postprocessing and error estimates, RAIRO Modél. Math. Anal. Numér., 19 (1985), pp. 7-32. 
[13] J. P. Aubin And H. G. Burchard, Some aspects of the method of the hypercircle applied to elliptic variational problems, in Numerical Solution of Partial Differential Equations, II (SYNSPADE 1970) (Proc. Sympos., Univ. of Maryland, College Park, Md., 1970), Academic Press, New York, 1971, pp. 167.

[14] I. BabušKa And T. Strouboulis, The finite element method and its reliability, Numerical Mathematics and Scientific Computation, The Clarendon Press Oxford University Press, New York, 2001.

[15] I. BabušKa, T. Strouboulis, and S. K. Gangaraj, Guaranteed computable bounds for the exact error in the finite element solution. I. One-dimensional model problem, Comput. Methods Appl. Mech. Engrg., 176 (1999), pp. 51-79. New advances in computational methods (Cachan, 1997).

[16] P. Bastian and B. RivièRe, Superconvergence and $H($ div) projection for discontinuous Galerkin methods, Internat. J. Numer. Methods Fluids, 42 (2003), pp. 1043-1057.

[17] M. Bebendorf, A note on the Poincaré inequality for convex domains, Z. Anal. Anwendungen, 22 (2003), pp. 751-756.

[18] D. Braess, V. Pillwein, And J. SchöBerl, Equilibrated residual error estimates are p-robust, Comput. Methods Appl. Mech. Engrg., 198 (2009), pp. 1189-1197.

[19] D. Braess And J. SchöBerl, Equilibrated residual error estimator for edge elements, Math. Comp., 77 (2008), pp. 651-672.

[20] S. C. Brenner, Poincaré-Friedrichs inequalities for piecewise $H^{1}$ functions, SIAM J. Numer. Anal., 41 (2003), pp. 306-324.

[21] F. Brezzi And M. Fortin, Mixed and hybrid finite element methods, vol. 15 of Springer Series in Computational Mathematics, Springer-Verlag, New York, 1991.

[22] C. Carstensen, A unifying theory of a posteriori finite element error control, Numer. Math., 100 (2005), pp. 617-637.

[23] C. Carstensen, M. Eigel, R. H. W. Hoppe, and C. Löbhard, A review of unified a posteriori finite element error control, Numer. Math. Theory Methods Appl., 5 (2012), pp. 509-558.

[24] C. Carstensen and S. A. Funken, Fully reliable localized error control in the FEM, SIAM J. Sci. Comput., 21 (1999/00), pp. 1465-1484.

[25] C. Carstensen and S. A. Funken, Constants in Clément-interpolation error and residual based a posteriori error estimates in finite element methods, East-West J. Numer. Math., 8 (2000), pp. 153-175.

[26] C. Carstensen, T. Gudi, and M. Jensen, A unifying theory of a posteriori error control for discontinuous Galerkin FEM, Numer. Math., 112 (2009), pp. 363-379.

[27] C. Carstensen and J. Hu, A unifying theory of a posteriori error control for nonconforming finite element methods, Numer. Math., 107 (2007), pp. 473-502.

[28] C. Carstensen and C. Merdon, Estimator competition for Poisson problems, J. Comput. Math., 28 (2010), pp. 309-330.

[29] Computational survey on a posteriori error estimators for nonconforming finite element methods for the Poisson problem, J. Comput. Appl. Math., 249 (2013), pp. 74-94.

[30] I. Cheddadi, R. Fučík, M. I. Prieto, And M. Vohralík, Computable a posteriori error estimates in the finite element method based on its local conservativity: improvements using local minimization, in CEMRACS 2007, vol. 24 of ESAIM Proc., EDP Sci., Les Ulis, 2008, pp. 77-96.

[31] P. G. Ciarlet, The Finite Element Method for Elliptic Problems, vol. 4 of Studies in Mathematics and its Applications, North-Holland, Amsterdam, 1978. 
[32] S. Cochez-Dhondt And S. Nicaise, Equilibrated error estimators for discontinuous Galerkin methods, Numer. Methods Partial Differential Equations, 24 (2008), pp. 1236-1252.

[33] M. Costabel, M. Dauge, and L. Demkowicz, Polynomial extension operators for $H^{1}$, $H$ (curl) and $H$ (div)-spaces on a cube, Math. Comp., 77 (2008), pp. 1967-1999.

[34] M. Costabel And A. McIntosh, On Bogovskil and regularized Poincaré integral operators for de Rham complexes on Lipschitz domains, Math. Z., 265 (2010), pp. 297-320.

[35] E. Dari, R. Durán, C. PAdRa, And V. VAmpa, A posteriori error estimators for nonconforming finite element methods, RAIRO Modél. Math. Anal. Numér., 30 (1996), pp. 385-400.

[36] L. Demkowicz, J. Gopalakrishnan, and J. Schöberl, Polynomial extension operators. Part III, Math. Comp., 81 (2012), pp. 1289-1326.

[37] P. Destuynder And B. MÉTivet, Explicit error bounds for a nonconforming finite element method, SIAM J. Numer. Anal., 35 (1998), pp. 2099-2115.

[38] — Explicit error bounds in a conforming finite element method, Math. Comp., 68 (1999), pp. 13791396.

[39] D. A. Di Pietro And A. Ern, Mathematical Aspects of Discontinuous Galerkin Methods, vol. 69 of Mathématiques \& Applications, Springer-Verlag, Berlin, 2011.

[40] V. DoLEJší, Semi-implicit interior penalty discontinuous Galerkin methods for viscous compressible flows, Commun. Comput. Phys., 4 (2008), pp. 231-274.

[41] A. ERn, S. Nicaise, ANd M. Vohralík, An accurate $\mathbf{H}($ div) flux reconstruction for discontinuous Galerkin approximations of elliptic problems, C. R. Math. Acad. Sci. Paris, 345 (2007), pp. 709-712.

[42] A. Ern, A. F. Stephansen, And M. Vohralík, Guaranteed and robust discontinuous Galerkin a posteriori error estimates for convection-diffusion-reaction problems, J. Comput. Appl. Math., 234 (2010), pp. 114-130.

[43] A. ERn AND M. Vohralík, Flux reconstruction and a posteriori error estimation for discontinuous Galerkin methods on general nonmatching grids, C. R. Math. Acad. Sci. Paris, 347 (2009), pp. 441-444.

[44] _ A posteriori error estimation based on potential and flux reconstruction for the heat equation, SIAM J. Numer. Anal., 48 (2010), pp. 198-223.

[45] — Adaptive inexact Newton methods with a posteriori stopping criteria for nonlinear diffusion PDEs, SIAM J. Sci. Comput., 35 (2013), pp. A1761-A1791.

[46] _ - Four closely related equilibrated flux reconstructions for nonconforming finite elements, C. R. Math. Acad. Sci. Paris, 351 (2013), pp. 77-80.

[47] R. Eymard, T. Gallouët, and R. Herbin, Convergence of finite volume schemes for semilinear convection diffusion equations, Numer. Math., 82 (1999), pp. 91-116.

[48] — Finite volume methods, in Handbook of Numerical Analysis, Vol. VII, North-Holland, Amsterdam, 2000, pp. 713-1020.

[49] A. Hannukainen, R. Stenberg, and M. Vohralík, A unified framework for a posteriori error estimation for the Stokes problem, Numer. Math., 122 (2012), pp. 725-769.

[50] I. HlaváČek, J. Haslinger, J. NeČas, and J. Lovíšek, Solution of variational inequalities in mechanics, vol. 66 of Applied Mathematical Sciences, Springer-Verlag, New York, 1988. Translated from the Slovak by J. Jarník.

[51] D. W. KeLLy, The self-equilibration of residuals and complementary a posteriori error estimates in the finite element method, Internat. J. Numer. Methods Engrg., 20 (1984), pp. 1491-1506. 
[52] K.-Y. KIM, A posteriori error analysis for locally conservative mixed methods, Math. Comp., 76 (2007), pp. $43-66$.

[53] — A posteriori error estimators for locally conservative methods of nonlinear elliptic problems, Appl. Numer. Math., 57 (2007), pp. 1065-1080.

[54] — Guaranteed a posteriori error estimator for mixed finite element methods of elliptic problems, Appl. Math. Comput., 218 (2012), pp. 11820-11831.

[55] P. LAdevèze, Comparaison de modèles de milieux continus, Ph.D. thesis, Université Pierre et Marie Curie (Paris 6), 1975.

[56] P. LAdevèze AND D. Leguillon, Error estimate procedure in the finite element method and applications, SIAM J. Numer. Anal., 20 (1983), pp. 485-509.

[57] R. Luce And B. I. Wohlmuth, A local a posteriori error estimator based on equilibrated fluxes, SIAM J. Numer. Anal., 42 (2004), pp. 1394-1414.

[58] J. M. Melenk and B. I. Wohlmuth, On residual-based a posteriori error estimation in hp-FEM, Adv. Comput. Math., 15 (2001), pp. 311-331 (2002). A posteriori error estimation and adaptive computational methods.

[59] S. Nicaise, K. Witowski, and B. I. Wohlmuth, An a posteriori error estimator for the Lamé equation based on equilibrated fluxes, IMA J. Numer. Anal., 28 (2008), pp. 331-353.

[60] C. PADRA, A posteriori error estimators for nonconforming approximation of some quasi-Newtonian flows, SIAM J. Numer. Anal., 34 (1997), pp. 1600-1615.

[61] N. PARÉs, P. DíEz, And A. Huerta, Subdomain-based flux-free a posteriori error estimators, Comput. Methods Appl. Mech. Engrg., 195 (2006), pp. 297-323.

[62] N. Parés, H. SAntos, And P. DíEz, Guaranteed energy error bounds for the Poisson equation using a flux-free approach: solving the local problems in subdomains, Internat. J. Numer. Methods Engrg., 79 (2009), pp. 1203-1244.

[63] L. E. Payne And H. F. Weinberger, An optimal Poincaré inequality for convex domains, Arch. Rational Mech. Anal., 5 (1960), pp. 286-292.

[64] W. Prager And J. L. Synge, Approximations in elasticity based on the concept of function space, Quart. Appl. Math., 5 (1947), pp. 241-269.

[65] S. Prudhomme, F. Nobile, L. Chamoin, and J. T. Oden, Analysis of a subdomain-based error estimator for finite element approximations of elliptic problems, Numer. Methods Partial Differential Equations, 20 (2004), pp. 165-192.

[66] S. I. REPIN, A posteriori error estimation for nonlinear variational problems by duality theory, Zap. Nauchn. Sem. S.-Peterburg. Otdel. Mat. Inst. Steklov. (POMI), 243 (1997), pp. 201-214, 342.

[67] — A posteriori error estimation for variational problems with uniformly convex functionals, Math. Comp., 69 (2000), pp. 481-500.

[68] — A posteriori estimates for partial differential equations, vol. 4 of Radon Series on Computational and Applied Mathematics, Walter de Gruyter GmbH \& Co. KG, Berlin, 2008.

[69] J. E. Roberts And J.-M. Thomas, Mixed and hybrid methods, in Handbook of Numerical Analysis, Vol. II, North-Holland, Amsterdam, 1991, pp. 523-639.

[70] I. ŠEBestová And T. VeJChODSkÝ, Two-sided bounds for eigenvalues of differential operators with applications to Friedrichs, Poincaré, trace, and similar constants, SIAM J. Numer. Anal., 52 (2014), pp. 308-329. 
[71] G. Stoyan and Á. Baran, Crouzeix-Velte decompositions for higher-order finite elements, Comput. Math. Appl., 51 (2006), pp. 967-986.

[72] J. L. Synge, The hypercircle in mathematical physics: a method for the approximate solution of boundary value problems, Cambridge University Press, New York, 1957.

[73] A. Veeser and R. Verfürth, Poincaré constants for finite element stars, IMA J. Numer. Anal., 32 (2012), pp. 30-47.

[74] R. Verfürth, A posteriori error estimation techniques for finite element methods, Numerical Mathematics and Scientific Computation, Oxford University Press, Oxford, 2013.

[75] M. Vohralík, On the discrete Poincaré-Friedrichs inequalities for nonconforming approximations of the Sobolev space $H^{1}$, Numer. Funct. Anal. Optim., 26 (2005), pp. 925-952.

[76] — A posteriori error estimates for lowest-order mixed finite element discretizations of convectiondiffusion-reaction equations, SIAM J. Numer. Anal., 45 (2007), pp. 1570-1599.

[77] _ A posteriori error estimation in the conforming finite element method based on its local conservativity and using local minimization, C. R. Math. Acad. Sci. Paris, 346 (2008), pp. 687-690.

[78] — Unified primal formulation-based a priori and a posteriori error analysis of mixed finite element methods, Math. Comp., 79 (2010), pp. 2001-2032.

[79] - Guaranteed and fully robust a posteriori error estimates for conforming discretizations of diffusion problems with discontinuous coefficients, J. Sci. Comput., 46 (2011), pp. 397-438. 